ARTICLE

\title{
Neuronal and glial 3D chromatin architecture informs the cellular etiology of brain disorders
}

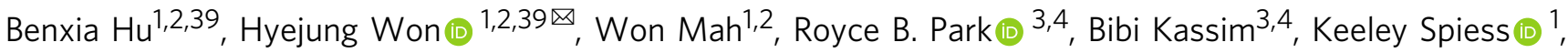 \\ Alexey Kozlenkov,4,5, Cheynna A. Crowley (10 ${ }^{2}$, Sirisha Pochareddy ${ }^{6}$, The PsychENCODE Consortium*, \\ Yun Li2 $2,7,8$, Stella Dracheva $3,4,5$, Nenad Sestan (1D) 9,10,11,12, Schahram Akbarian (iD ${ }^{3,4}$ \& \\ Daniel H. Geschwind (13,14,15,16凶
}

Cellular heterogeneity in the human brain obscures the identification of robust cellular regulatory networks, which is necessary to understand the function of non-coding elements and the impact of non-coding genetic variation. Here we integrate genome-wide chromosome conformation data from purified neurons and glia with transcriptomic and enhancer profiles, to characterize the gene regulatory landscape of two major cell classes in the human brain. We then leverage cell-type-specific regulatory landscapes to gain insight into the cellular etiology of several brain disorders. We find that Alzheimer's disease (AD)-associated epigenetic dysregulation is linked to neurons and oligodendrocytes, whereas genetic risk factors for AD highlighted microglia, suggesting that different cell types may contribute to disease risk, via different mechanisms. Moreover, integration of glutamatergic and GABAergic regulatory maps with genetic risk factors for schizophrenia (SCZ) and bipolar disorder (BD) identifies shared (parvalbumin-expressing interneurons) and distinct cellular etiologies (upper layer neurons for BD, and deeper layer projection neurons for SCZ). Collectively, these findings shed new light on cell-type-specific gene regulatory networks in brain disorders.

\footnotetext{
${ }^{1}$ UNC Neuroscience Center, University of North Carolina, Chapel Hill, NC, USA. ${ }^{2}$ Department of Genetics, University of North Carolina, Chapel Hill, NC, USA.

${ }^{3}$ Friedman Brain Institute, Icahn School of Medicine at Mount Sinai, New York, NY, USA. ${ }^{4}$ Department of Psychiatry, Icahn School of Medicine at Mount Sinai, New York, NY, USA. ${ }^{5}$ James J. Peters VA Medical Center, Bronx, NY, USA. ${ }^{6}$ Department of Neuroscience and Kavli Institute for Neuroscience, Yale School of Medicine, New Haven, CT, USA. ${ }^{7}$ Biostatistics, University of North Carolina, Chapel Hill, NC, USA. ${ }^{8}$ Computer Science, University of North Carolina, Chapel Hill, NC, USA. ${ }^{9}$ Department of Psychiatry, Yale School of Medicine, New Haven, CT, USA. ${ }^{10}$ Department of Genetics, Yale School of Medicine, New Haven, CT, USA. ${ }^{11}$ Department of Comparative Medicine, Program in Integrative Cell Signaling and Neurobiology of Metabolism, Yale School of Medicine, New Haven, CT, USA. ${ }^{12}$ Program in Cellular Neuroscience, Neurodegeneration, and Repair and Yale Child Study Center, Yale School of Medicine, New Haven, CT, USA. ${ }^{13}$ Neurogenetics Program, Department of Neurology, David Geffen School of Medicine University of California, Los Angeles, CA, USA. ${ }^{14}$ Center for Autism Research and Treatment, Semel Institute, David Geffen School of Medicine University of California, Los Angeles, CA 90095, USA. ${ }^{15}$ Department of Human Genetics, David Geffen School of Medicine University of California, Los Angeles, CA, USA. ${ }^{16}$ Department of Psychiatry and Biobehavioral Sciences, Semel Institute, David Geffen School of Medicine University of California, Los Angeles, CA, USA. ${ }^{39}$ These authors contributed equally: Benxia Hu, Hyejung Won. ${ }^{\star} A$ list of authors and their affiliations appears at the end of the paper. ${ }^{凶}$ email: hyejung_won@med.unc.edu; dhg@mednet.ucla.edu
} 
T he majority of human genetic variants imparting risk for brain diseases are located within non-coding elements ${ }^{1}$. Allelic variation in these elements is thought to have an influence on complex human traits via impacting gene regulation ${ }^{2}$, necessitating the understanding of gene regulatory architecture in the human brain. We and others have identified gene regulatory relationships in the developing and adult human brain by integrating multi-dimensional datasets that include transcriptomic, epigenomic, and higher-order chromatin interaction landscapes ${ }^{3-7}$. However, cellular heterogeneity poses significant challenges for unraveling the complexity in the gene regulatory architecture of the human brain, as it is comprised of heterogeneous cell populations, mostly different types of neurons and glia, each of which display distinct gene expression ${ }^{3,8-10}$ and chromatin accessibility profiles ${ }^{1-14}$. To address this issue, several groups have employed $\mathrm{Hi}-\mathrm{C}$ and its derivatives (e.g. promotercapture $\mathrm{Hi}-\mathrm{C}$ ) to build higher-order chromatin interaction maps in induced pluripotent stem cells (iPSC)-derived neurons and astrocytes ${ }^{15}$. However, these published studies rely on in vitro cultured cells that mark early brain development. Recently, promoter interaction profiles were inferred from three types of brain cells (neurons, oligodendrocytes, and microglia) obtained from the postnatal human cerebral cortex ${ }^{16}$.

Here, we analyze genome-wide chromosome conformation at cellular resolution to capture how chromatin structure affects cellular expression profiles. We use fluorescence-activated nuclear sorting (FANS, "Methods") ${ }^{17}$ to sort neurons (NeuN+ cells) and glia (NeuN- cells), two major cell classes in the brain, and generated genome-wide chromosome conformation using Hi-C (Supplementary Figs. 1-2). We call multiple architectural units: A/B compartments, which are mutually exclusive large-scale chromatin domains, where compartment $\mathrm{A}$ is associated with actively transcribed regions and compartment $\mathrm{B}$ is enriched with repressive chromatin regions; Topologically Associating Domains (TADs), which are enriched with self-contacts and thus delineate the genomic regions within which most cis-regulatory interactions occur; Frequently Interacting REgions (FIREs), which are local interaction hotspots that are enriched with active regulatory elements; and gene loops within $\mathrm{NeuN}+$ and NeuN- cells which link promotors to presumed regulatory elements (Supplementary Fig. 1). Furthermore, we integrate acetylated histone $\mathrm{H} 3$ lysine 27 (H3K27ac) peaks from glutamatergic (Glu) and medial ganglionic eminence (MGE)-derived GABAergic (GABA) neurons ${ }^{18}$ with $\mathrm{NeuN}+$ chromatin interactions to obtain finer-scale gene regulatory relationship within these two major neuronal subtypes. We then leveraged cell-type-specific gene regulatory relationships to help decipher the genetic mechanisms contributing to Alzheimer's disease (AD), schizophrenia (SCZ), and bipolar disorder (BD). Our results illustrate deciphering the epigenetic landscape in a cell-type-specific fashion offers substantial advantages for inferring the functional impact of genetic risk factors associated with brain disorders.

\section{Results}

Differential FIREs and super-FIREs are associated with celltype-specific gene regulation. Hi-C libraries were generated from $\mathrm{NeuN}+$ and $\mathrm{NeuN}-$ cells sorted from four dorsolateral prefrontal cortex (DLPFC) samples (Supplementary Table 1 and Supplementary Fig. 2). We first measured the reproducibility across Hi-C libraries using a stratum-adjusted correlation coefficient (SCC) that systematically quantifies similarities between two $\mathrm{Hi}-\mathrm{C}$ contact maps ${ }^{19}$. Notably, we found that samples were clustered by cell types, not individuals, and showed high correlation between the same cell types from different individuals $(\mathrm{NeuN}+=0.95-0.97$; NeuN $-=0.86-0.91$; Supplementary Fig. 3a), demonstrating the reproducibility across Hi-C libraries.
We also compared the chromatin contacts of NeuN+ and $\mathrm{NeuN}$ - cells with other existing $\mathrm{Hi}-\mathrm{C}$ datasets from brainrelevant tissues (Supplementary Fig. 3b, "Methods"). We found that NeuN- cells showed higher structural similarity with the adult brain than the fetal brain, indicative of gliogenesis in the postnatal brain ${ }^{20}$. Intriguingly, NeuN- cells did not show high structural similarity with iPSC-derived astrocytes, consistent with the previous report that the majority of $\mathrm{NeuN}-$ cells are oligodendrocytes $^{21}$. NeuN + cells showed high similarity with adult brains, fetal brains, and iPSC-derived neurons. The fact that NeuN + cells show high similarity with fetal brain may reflect extensive neurogenesis at midgestation, the developmental stage at which the fetal brain was obtained ${ }^{22}$.

We next interrogated the cell-type-specific nature of 3D chromatin structures such as compartments ${ }^{23}$ and FIREs ${ }^{6,24}$. We detected extensive compartment switching between NeuN+ and NeuN- cells: 4,333 regions (in 100kb resolution) switched from compartment A to B between NeuN- to NeuN+ cells, while 2098 regions switched from compartment $\mathrm{B}$ to $\mathrm{A}$ in $\mathrm{NeuN}+$ to $\mathrm{NeuN}-$ cells. Importantly, genes located in compartments that switch from A to B between NeuN- and NeuN+ were highly expressed in oligodendrocytes and astrocytes, while those that switch from B to A in NeuN- to NeuN+ were highly expressed in neurons, suggesting that the difference in chromosome conformation between NeuN+ and $\mathrm{NeuN}-$ cells is associated with cell-type-specific gene regulation (Supplementary Fig. 3c).

FIREs represent regions that act as interaction hubs, containing active enhancers that show high tissue specificity ${ }^{6}$. They are also enriched for genome-wide association study (GWAS) variants and exhibit variability across individuals ${ }^{25,26}$. We therefore compared FIREs in NeuN+ and NeuN- cells to identify how local chromatin architecture differs among these major brain cell types. We detected 3966 and 3967 FIREs in NeuN+ and NeuN- cells, respectively (Fig. 1a and Supplementary Data 1$)^{24}$. Among them, 1248 FIREs were shared between NeuN + and $\mathrm{NeuN}-$ cells based on the coordinate-level overlap and similarities in FIRE scores (hereafter referred to as common FIREs, "Methods", Supplementary Data 1). Genes located in common FIREs were involved in housekeeping functions (e.g., microtubule polymerization, cell junction assembly) as well as pathways that involve both neurons and oligodendrocytes (e.g., main axon, Supplementary Data 1).

To further investigate how FIREs are associated with cell-typespecific gene expression, we used a stringent statistical cutoff to define differential FIREs on the basis of FIRE scores ("Methods"), detecting 287 differential FIREs between NeuN+ (145) and NeuN - (142) cells (hereby referred to as NeuN+ and NeuN- FIREs, respectively, Fig. 1b, Supplementary Data 2). Since previous reports have suggested that FIREs are closely linked to cell-typespecific enhancers ${ }^{6}$, we intersected differential FIREs with differential H3K27ac peaks between NeuN + and NeuN- cells ${ }^{27}$. We found that the majority of NeuN+ and NeuN- FIREs overlapped with NeuN+ and NeuN- differential H3K27ac peaks, respectively, displaying remarkable cell-type-specificity (Fig. 1c, d). We next examined whether differential FIREs overlap with celltype-specific marker genes. Indeed, NeuN+ FIREs overlapped with neuronal genes that were enriched for synaptic function (Fig. 1g), while NeuN- FIREs overlapped with genes involved in myelination, glial differentiation, and oligodendrocyte differentiation (Fig. 1h). Salient examples include GRIN2B, which overlaps with a NeuN+ FIRE, and OLIG1 and OLIG2, which overlap with a NeuN- FIRE (Fig. 1e, f, Supplementary Fig. 4). We further checked cellular expression profiles of genes assigned to differential FIREs using single-cell (sc)RNA-seq data ${ }^{10}$. As expected, NeuN+ and NeuN- FIRE-associated genes were mainly enriched in neurons and glia, respectively (Fig. li, j). In particular, NeuN- FIRE-associated genes were most highly expressed in 
a

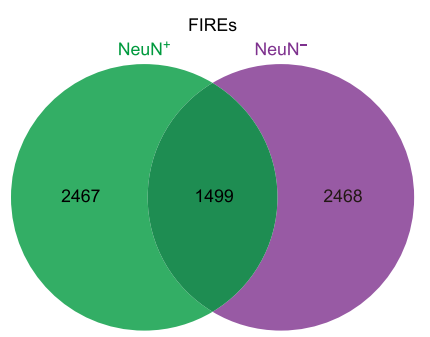

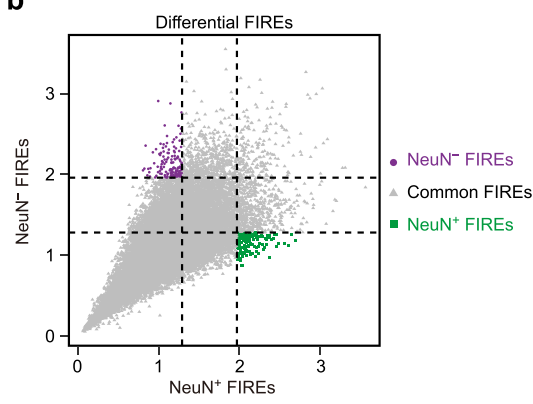

C

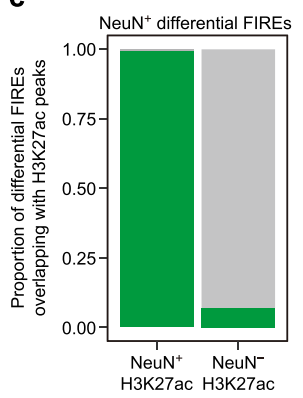

d

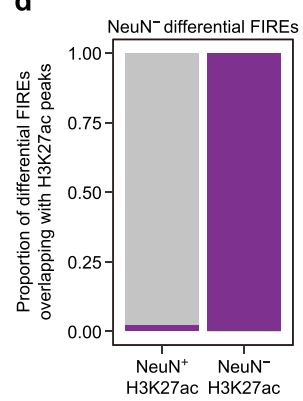

e
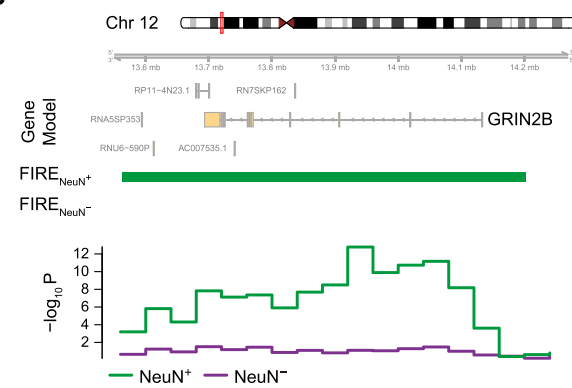

g

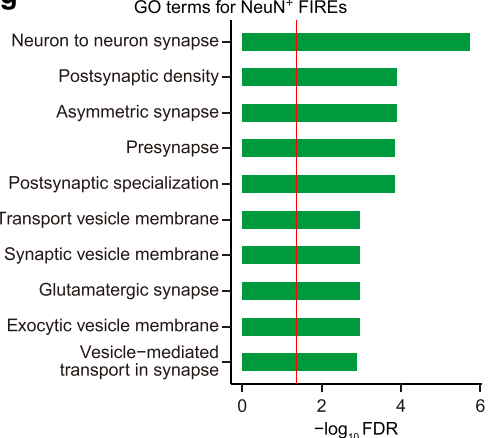

h

h

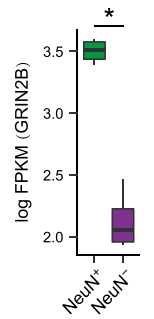

f
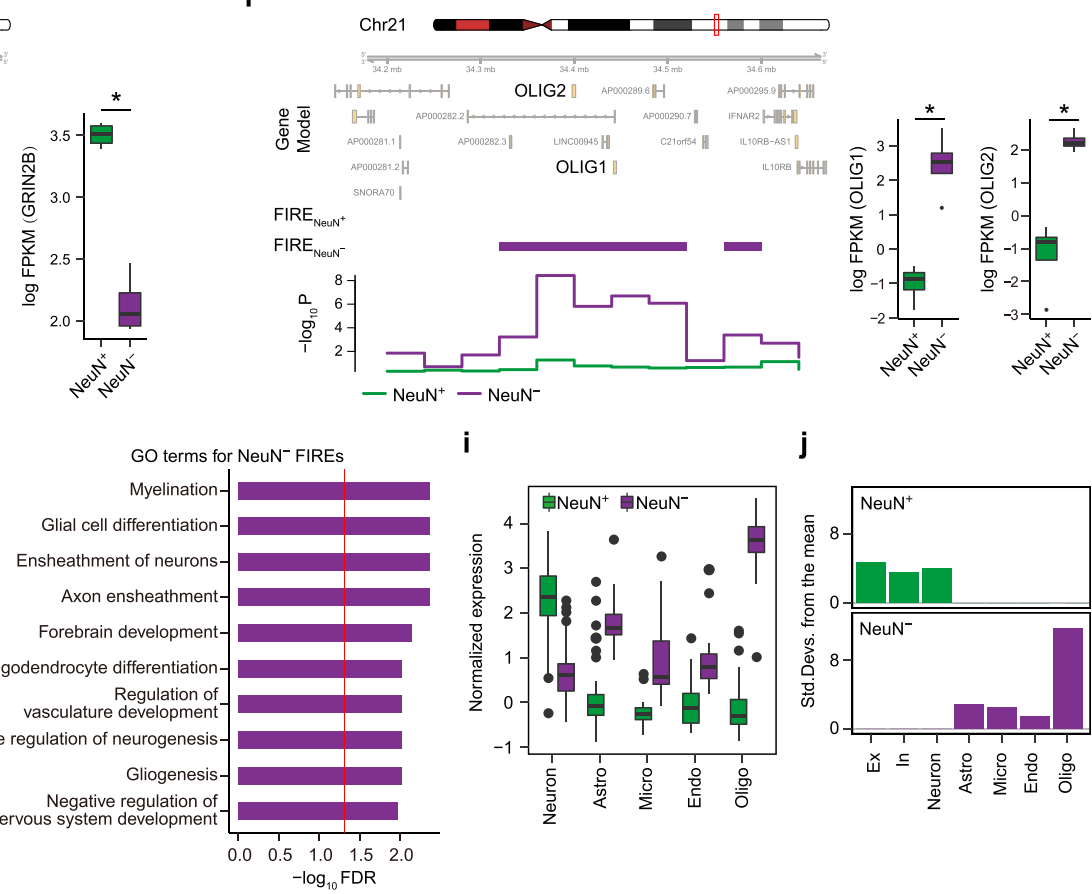

i

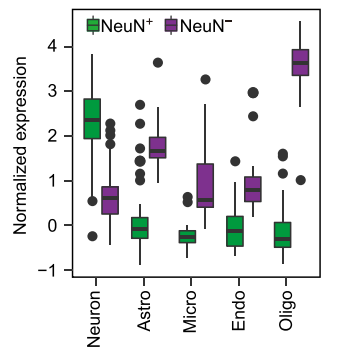

j

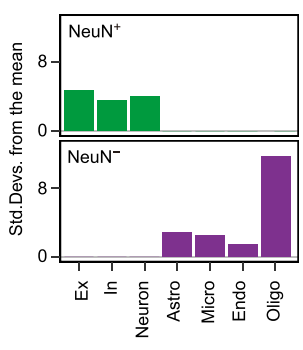

Fig. 1 Differential FIREs are associated with cell-type-specific gene regulation. a The number of FIREs detected in NeuN+and NeuN-cells. b Differential FIREs were identified in NeuN + and NeuN - cells. c, d Differential FIREs overlap with differential H3K27ac peaks in the corresponding cell types. A neuronal gene, GRIN2B, is located in NeuN+ specific FIREs (e), while two oligodendrocytic genes, OLIG1 and OLIG2, are located in NeuN- specific FIREs (f). FIREs and significance of FIRE scores in NeuN+ and NeuN- cells are depicted in green and purple, respectively. Boxplots in the right show expression levels of GRIN2B $(F D R=3.71 \mathrm{e}-12), \mathrm{OLIG1}(\mathrm{FDR}=7.34 \mathrm{e}-26)$ and OLIG2 (FDR $=8.06 \mathrm{e}-23)$ in NeuN+ $(n=4)$ and NeuN $-(n=4)$ cells. FPKM Fragments Per Kilobase of transcript per Million mapped reads. Center, median; box = 1st to 3rd quartiles (Q); minima, Q1 - $1.5 \times$ interquartile range (IQR); maxima, Q3 + $1.5 \times 1 \mathrm{IQR}$. ${ }^{\star}$ FDR $<0.05$ calculated by DESeq2 (two-sided Wald test). Source data are provided as a Source Data file. Gene ontology (GO) analysis for genes assigned to differential NeuN+ (g) and NeuN- (h) FIREs. The red line denotes FDR $=0.05$. i Cellular expression levels of genes assigned to differential NeuN+ and NeuN- FIREs. Center, median; box =Q1-Q3; minima, Q1 - $1.5 \times$ IQR; maxima, Q3 + $1.5 \times$ IQR. Neurons, $n=131$; astrocytes (Astro), $n=62 ;$ microglia (Micro), $n=16$; endothelial (Endo), $n=20$; oligodendrocytes (Oligo), $n=38$. Source data are provided as a Source Data file. $\mathbf{j}$ Genes assigned to differential $\mathrm{NeuN}+$ and NeuN- FIREs are enriched in neurons and glia, respectively. Ex excitatory neurons, In inhibitory neurons.

oligodendrocytes, confirming that NeuN- fraction is enriched for oligodendrocytes ${ }^{21}$.

Super-FIREs represent a small proportion of FIRE clusters with the most significant local interaction frequency ${ }^{6}$. Super-FIREs are thought to have a strong gene regulatory potential and often overlap with super enhancers ${ }^{6}$. We therefore identified super-FIREs in NeuN+ and NeuN - cells ("Methods") and found that they also displayed substantial cellular specificities. Only 9 super-FIREs were detected in both NeuN+ and NeuN- cells, leaving 253 and 157 celltype-specific super-FIREs in NeuN+ and NeuN- cells, respectively (Supplementary Fig. 5a, Supplementary Data 2). Over 95\% of superFIREs overlapped with differential H3K27ac peaks and all superFIREs overlapped with promoters, indicating that they have a particularly strong cell-type-specific regulatory impact (Supplementary Fig. 5b, c). In line with these findings, super-FIREs were tightly coupled with cell-type-specific gene expression. NeuN+ superFIREs overlapped with genes functioning in synapses and ion gated channels that were highly expressed in neurons, while NeuNsuper-FIREs overlapped with genes involved in cell adhesion, myelination, and ensheathment of neurons, with high expression in glia (Supplementary Fig. 5d-f, 6). Taken together, these analyses show that differential FIREs and super-FIREs are strongly associated with cell-type-specific gene regulation in the nervous system.

Characteristics of chromatin interactions in $\mathrm{NeuN}+$ and $\mathrm{NeuN}-$ cells. We next identified promoter-anchored chromatin loops in $\mathrm{NeuN}+$ and NeuN- cells to examine how chromatin interactions are associated with intricate regulation of cell-type-specific expression profiles. We detected 187,674 and 167,551 promoter-based 
a

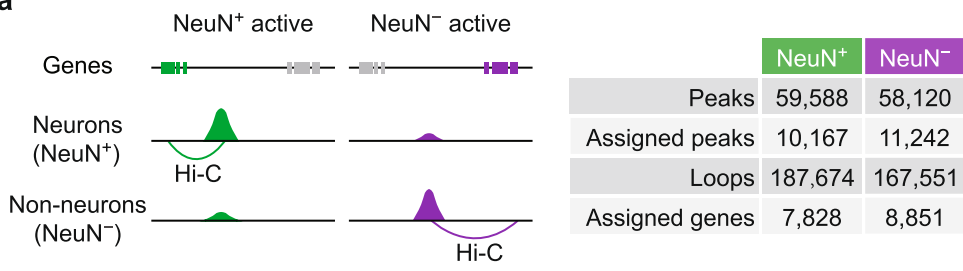

b
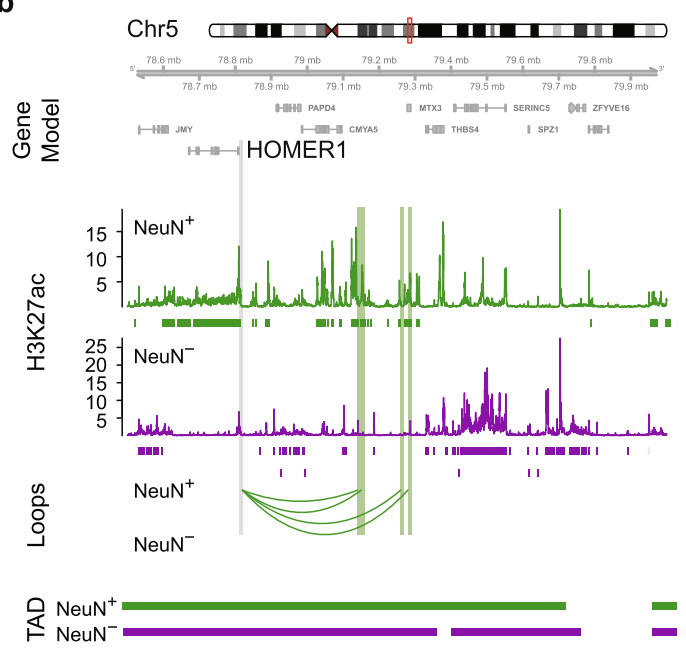

c
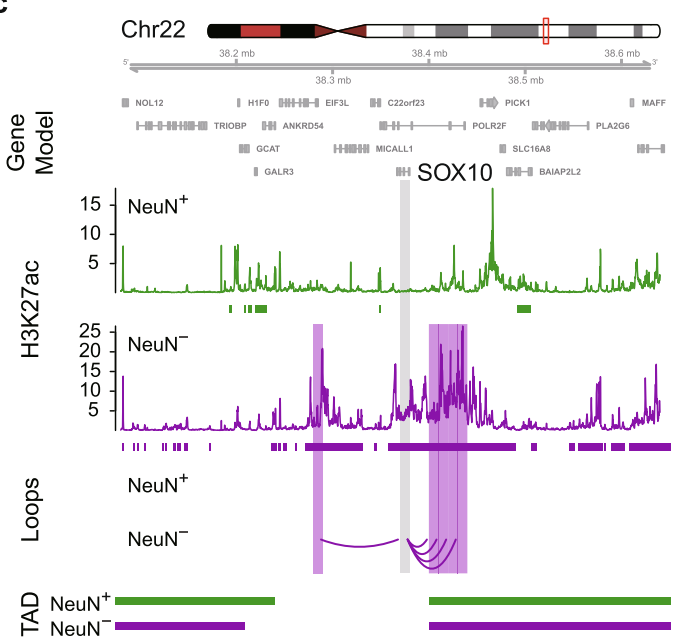

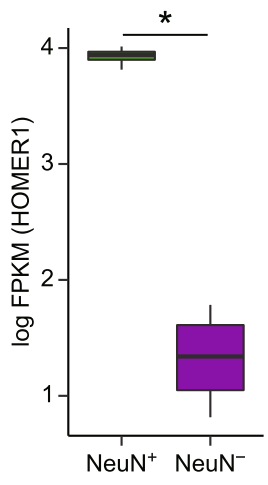

d
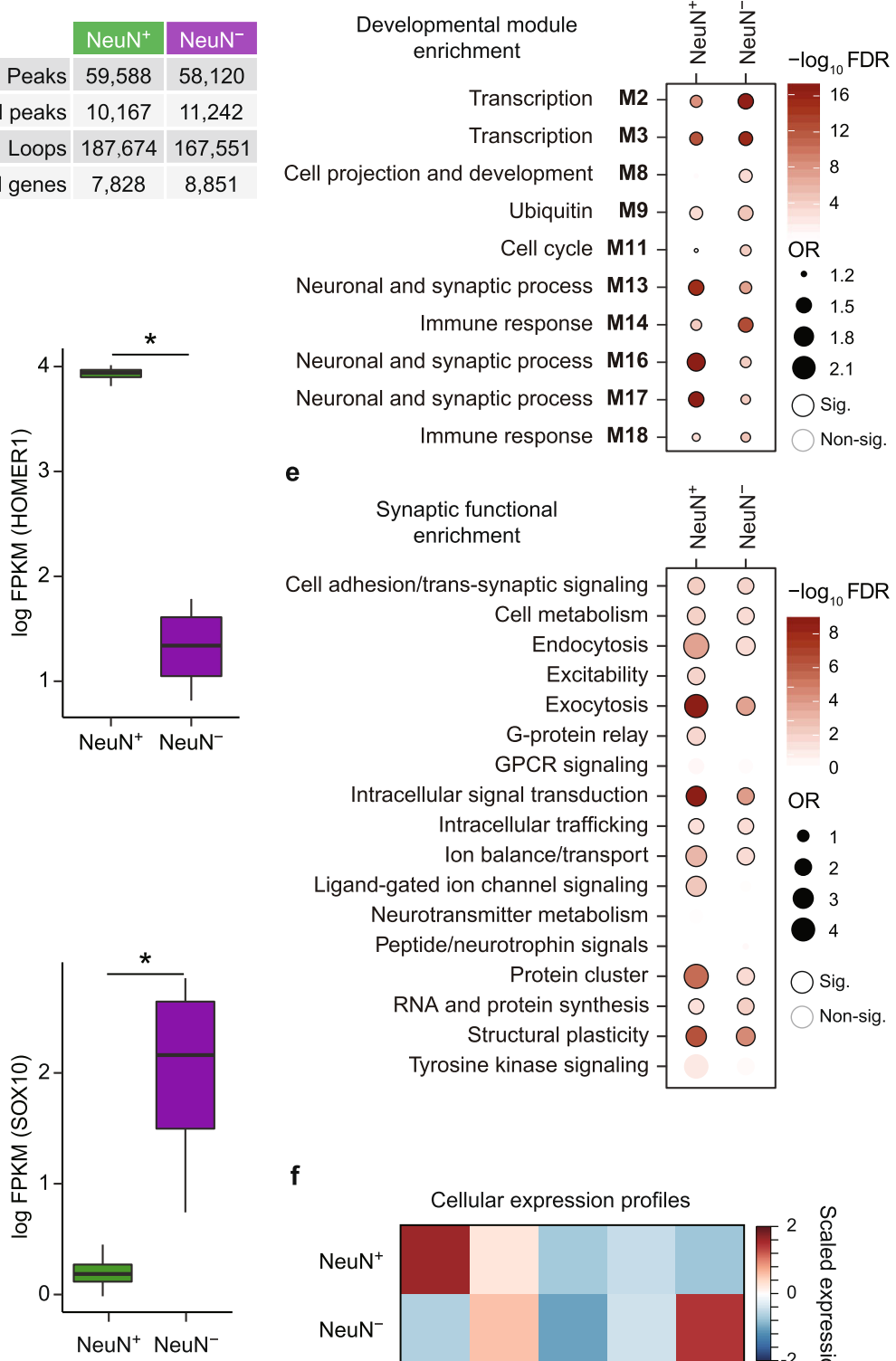

C

$$
\text { Neu }
$$

e

$\mathbf{f}$

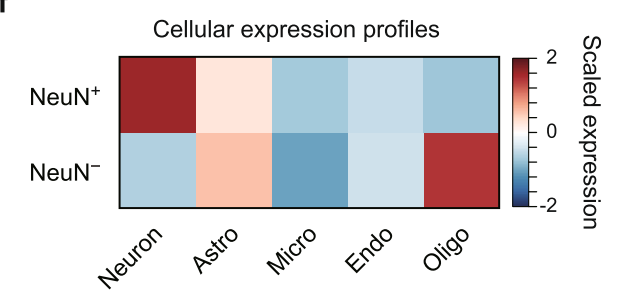

Fig. 2 Enhancer-promoter interactions in $\mathbf{N e u} \mathbf{N}+$ and $\mathbf{N e u} \mathbf{N}-$ cells. a (Left) cell-type-specific regulatory networks were built by linking genes to NeuN+ and $\mathrm{NeuN}$ - specific H3K27ac peaks via Hi-C interactions in $\mathrm{NeuN}+$ and NeuN- cells, respectively. (Right) The number of cell-type-specific peaks and their assigned genes in NeuN+ and NeuN- cells is described. A neuronal gene, HOMER1, is engaged with NeuN+ specific H3K27ac peaks via loops in $\mathrm{NeuN}+$ cells (b), while an oligodendrocyte gene, SOX10, is engaged with NeuN- specific H3K27ac peaks via loops in NeuN-cells (c). The regions that interact with the gene promoter (gray) are highlighted in green $(\mathrm{NeuN}+)$ and purple $(\mathrm{NeuN}-)$, respectively. Boxplots in the right show expression levels of HOMERT (FDR $=7.26 \mathrm{e}-32)$ and SOX10 (FDR $=1.88 \mathrm{e}-49)$ in NeuN $+(n=4)$ and NeuN- $(n=4)$ cells, respectively. FPKM Fragments Per Kilobase of transcript per Million mapped reads. Center, median; box =Q1-Q3; minima, Q1 - $1.5 \times$ IQR; maxima, Q3 + $1.5 \times I Q R$. *FDR $<0.05$ calculated by DESeq2 (two-sided Wald test). Source data are provided as a Source Data file. $\mathbf{d}$ Genes assigned to NeuN+ specific peaks are enriched for synaptic co-expression modules, while genes assigned to NeuN- specific peaks are enriched for co-expression modules involved in transcriptional regulation and immune response during neurodevelopment. Significant enrichment (Sig.), FDR $<0.05$. Fisher's exact test was used for statistics analysis. OR, odds ratio. e Genes assigned to NeuN+ specific peaks are more highly enriched for synaptic functions such as exocytosis, intracellular signal transduction, protein cluster and structural plasticity than genes assigned to NeuN- specific peaks. Sig., FDR $<0.05$. Fisher's exact test was used for statistics analysis. $\mathbf{f}$ Genes assigned to $\mathrm{NeuN}+$ specific peaks are highly expressed in neurons, while genes assigned to NeuN- specific peaks are highly expressed in oligodendrocytes and astrocytes. Astro astrocytes, Micro microglia, Endo Endothelial, Oligo oligodendrocytes.

interactions $^{3,4}$ from NeuN+ and NeuN-cells, respectively (Fig. 2a). Over $75 \%$ of promoter-based interactions were detected within TADs (Supplementary Fig. 7a). Roughly 37\% of interactions occurred between enhancers and promoters and $\sim 23 \%$ of interactions occurred between two different promoters (Supplementary
Fig. 7b). We infer that the promoter-promoter interactions are likely attributed to transcription factors (TFs) of coregulated genes. A substantial fraction of chromatin interactions were distal, as $50 \%$ of chromatin interactions brought two genomic regions linearly separated by $320 \mathrm{~kb}$ or more into close proximity (Supplementary 
Fig. 7c) and captured complex enhancer-promoter interactions. For example, we found that the majority of promoters interact with more than one enhancer (Supplementary Fig. 7d), consistent with the previous findings that multiple enhancers can interact with one promoter $^{3,7}$. Notably, the number of enhancers that interact with promoters was strikingly correlated with gene regulation, as gene expression linearly increased with the number of physically interacting enhancers up to a total of 10 or more (Supplementary Fig. $7 \mathrm{e})^{3,28}$.

We next leveraged chromatin states predicted by ChromHMM ${ }^{29}$ to delineate the epigenetic properties of the genomic regions that interact with promoters (Supplementary Fig. 7f). As expected, promoters often interact with active chromatin features such as other transcription start sites (TSS, 1_TssA and 2_TssAFlnk) and enhancers (6_EnhG and 7_Enh). However, it is of note that a significant proportion of promoters also interact with bivalent marks (10_TssBiv, 11_BivFlnk and 12_EnhBiv), suggesting that the associated regions are poised to be activated upon stimulation. Together, our results confirmed that gene expression was coordinately controlled by physical interactions of promoters with enhancers, indicative of transcriptional regulators that underlie celltype-specific gene regulation. So, we implemented GimmeMotifs ${ }^{30}$ to evaluate differential $\mathrm{TF}$ motif enrichment at cell-type-specific enhancers that interact with promoters in NeuN+ and NeuNcells. TFs involved in neuronal fate commitment, including ZBTB18, SMARCC1, TBR1, and NEUROD2, were enriched in NeuN+ cells (Supplementary Fig. 7g). Conversely, TF motifs for SOX2, SOX3, SOX4, SOX6, and SOX9 were enriched in NeuN- cells (Supplementary Fig. $7 \mathrm{~g}$ ). NeuN- distal enhancers were also enriched for motifs for the IRF family, such as IRF4, IRF7, IRF8, and IRF9 (Supplementary Fig. 7g), which contains key regulators of neural immune pathways expressed in glial cells ${ }^{31}$.

To further identify how enhancer-promoter interactions regulate cellular expression profiles, we next overlapped chromatin interactions with cell-type-specific enhancers ("Methods"). We were able to assign 10,167 and 11,242 cell-type-specific $\mathrm{H} 3 \mathrm{~K} 27 \mathrm{ac}$ peaks to 7828 and 8851 genes via chromatin interactions in NeuN+ and NeuN-cells, respectively (Fig. 2a, Supplementary Data 3). Linking cell-type-specific enhancers to cell-type-specific loops revealed specific aspects of chromatin architecture regulating cell-type-specific gene expression. For example, a gene that encodes a synaptic scaffolding protein, HOMER $1^{32}$, was engaged in NeuN+ specific peaks and loops and its expression was significantly higher in NeuN + cells compared with NeuN- cells (Fig. 2b). In contrast, a glial gene, SOX1033, was engaged in $\mathrm{NeuN}-$ specific $\mathrm{H} 3 \mathrm{~K} 27 \mathrm{ac}$ peaks and $\mathrm{Hi}-\mathrm{C}$ loops, and had higher expression in NeuN-cells than in NeuN+ cells (Fig. 2c). In line with these findings, NeuN+ enhancer-promoter interactions were enriched for synaptic and axonal genes, whereas $\mathrm{NeuN}$ - enhancer-promoter interactions were associated with actin-based motility (Supplementary Data 3). Moreover, genes assigned to $\mathrm{NeuN}+$ specific peaks were enriched in the synaptic co-expression modules during neurodevelopment ${ }^{34}$ (Fig. 2d). Within a synapse, they were involved in specialized functions including exocytosis, intracellular signal transduction, and synaptic plasticity ${ }^{35}$ (Fig. 2e). Lastly, genes assigned to NeuN+ specific peaks were more highly expressed in neurons, while those assigned to NeuN- specific peaks were highly expressed in oligodendrocytes and astrocytes (Fig. 2f), demonstrating the tight relationship between cell-type-specific chromatin architecture and expression signature.

Enhancer-promoter interactions in glutamatergic and GABAergic neurons. Single-cell expression profiles have demonstrated a remarkable transcriptional diversity within neuronal subtypes ${ }^{10}$. We therefore used H3K27ac peaks defined in purified Glu and MGE-derived GABA (hereafter referred to as GABA) neurons ${ }^{18}$ to deconvolute NeuN + chromatin interactions into two major neuronal subtypes. We obtained 45,911 Gluspecific and 32,169 GABA-specific H3K27ac peaks and assigned them to 6234 and 4342 genes, respectively (Supplementary Fig. 8a, Supplementary Data 4). These genes showed a remarked level of neuronal specificity. Genes assigned to Glu peaks were highly expressed in excitatory neurons, such as layers (L) $2 / 3$ pyramidal neurons (Ex1) and L5/6 corticothalamic projection neurons (Ex7), while genes assigned to GABA peaks were highly expressed in inhibitory neurons, such as parvalbumin-expressing GABA interneurons (In6, Supplementary Fig. 8b, the definition of Ex1-8 and In1-8 neuronal subtypes is available in Supplementary Table 2). GRIK4, a gene that encodes an ionotropic class of glutamate receptor ${ }^{36}$, displayed complex chromatin interactions with multiple Glu peaks. In contrast, GAD1, a gene that encodes a well-known cellular marker for inhibitory neurons ${ }^{37}$, was engaged in GABA peaks via chromatin interactions (Supplementary Fig. 8c). Collectively, we established neuronal subtypespecific gene regulatory relationships by integrating Glu- and GABA-specific peaks with the NeuN+ (neuronal) chromatin interaction profiles defined here.

Cell-type-specific nature of $\mathrm{AD}$-associated epigenetic dysregulation. We next used the cell-type-specific gene regulatory relationship to refine cell-type-specific aspects of disease vulnerability. Studies in $\mathrm{AD}$ have revealed changes in gene regulation manifested by differences in $\mathrm{H} 3 \mathrm{~K} 27 \mathrm{ac}$ in bulk tissue ${ }^{38}$, but how this relates to cell-type-specific vulnerability is not known. To this end, we attempted to deconvolve the cell-type-specificity of epigenetic dysregulation detected in the $\mathrm{AD}$ brain tissue ${ }^{38}$ by overlapping $\mathrm{AD}$-associated hyperacetylated and hypoacetylated H3K27ac peaks with $\mathrm{NeuN}+$ and NeuN- peaks (Fig. 3a). We detected that $\mathrm{AD}$-associated hyperacetylated peaks were largely active in NeuN-cells, while AD-associated hypoacetylated peaks were largely active in NeuN+ cells in neurotypical controls (Fig. 3b). To decipher the biological impact of AD-associated epigenetic dysregulation, we annotated these $\mathrm{AD}$-associated hyperacetylated and hypoacetylated H3K27ac peaks using chromatin interaction profiles. We were able to link hypoacetylated peaks in $\mathrm{AD}$ to 460 genes using $\mathrm{NeuN}+\mathrm{Hi}-\mathrm{C}$ data and hyperacetylated peaks in AD to 676 genes using NeuN- Hi-C data (hereby referred to as NeuN+ hypo- and NeuN- hyperacetylated genes, respectively, Fig. 3c, Supplementary Data 5). NeuN+ hypoacetylated genes include $C A C N G 3$, whose promoter formed a loop with a peak, which was preferentially active in NeuN+ cells and was hypoacetylated in postmortem AD brain. CACNG3 encodes a voltage-gated calcium channel ${ }^{39}$ and had significantly higher expression in neurotypical $\mathrm{NeuN}+$ cells compared to NeuN- cells (Fig. 3d). NeuN- hyperacetylated genes include EHD1 (Fig. 3e), a gene involved in endocytic recycling with another AD-associated gene $B I N 1^{40}$. EHD1 was both hyperacetylated and highly expressed in neurotypical NeuN- cells compared to NeuN+ cells. Importantly, the promoter of EHD1 formed a loop with the $\mathrm{AD}$ hyperacetylated peak that was preferentially active in NeuN-cells. GO analysis demonstrated that NeuN+ hypoacetylated genes included synaptic genes while NeuN - hyperacetylated genes were involved in catalytic activity and glycoprotein binding (Supplementary Data 5). Cellular expression profiles also confirmed these findings, as NeuN+ hypoacetylated genes were highly expressed in neurons, while NeuN - hyperacetylated genes were highly expressed in oligodendrocytes, astrocytes, and endothelial cells (Fig. 3f). These results collectively suggest that in $\mathrm{AD}$ brains, many neuronal 
a

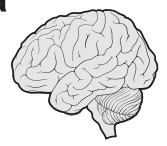

Control

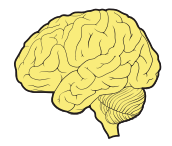

AD
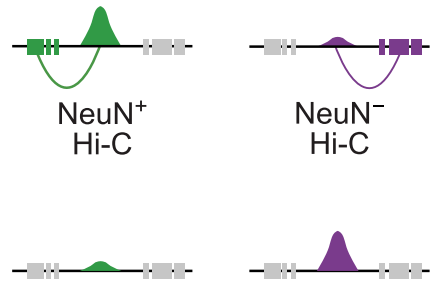

Hypoacetylation Hyperacetylation b

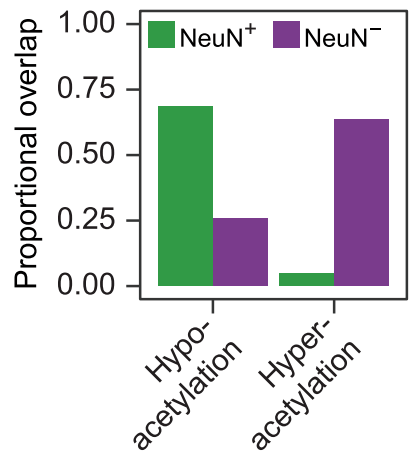

C

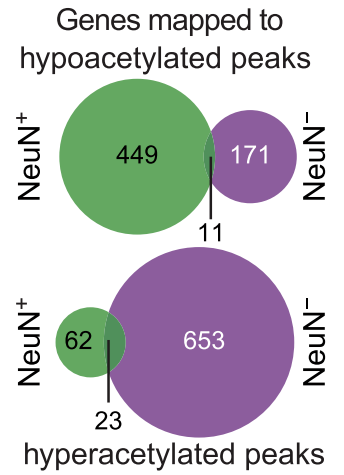

d
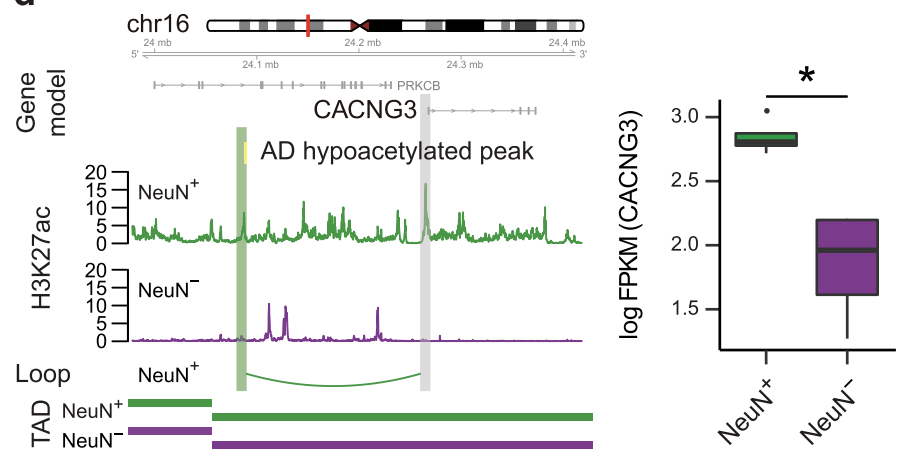

e
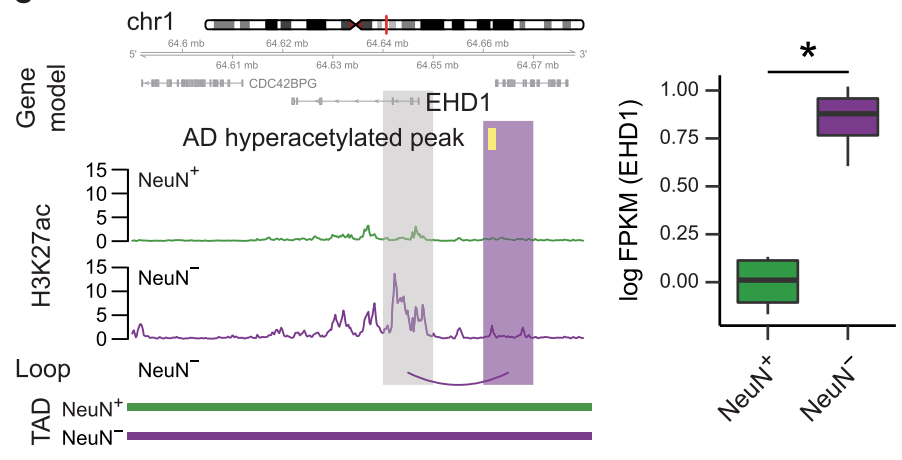

f

\section{Cellular expression profiles}
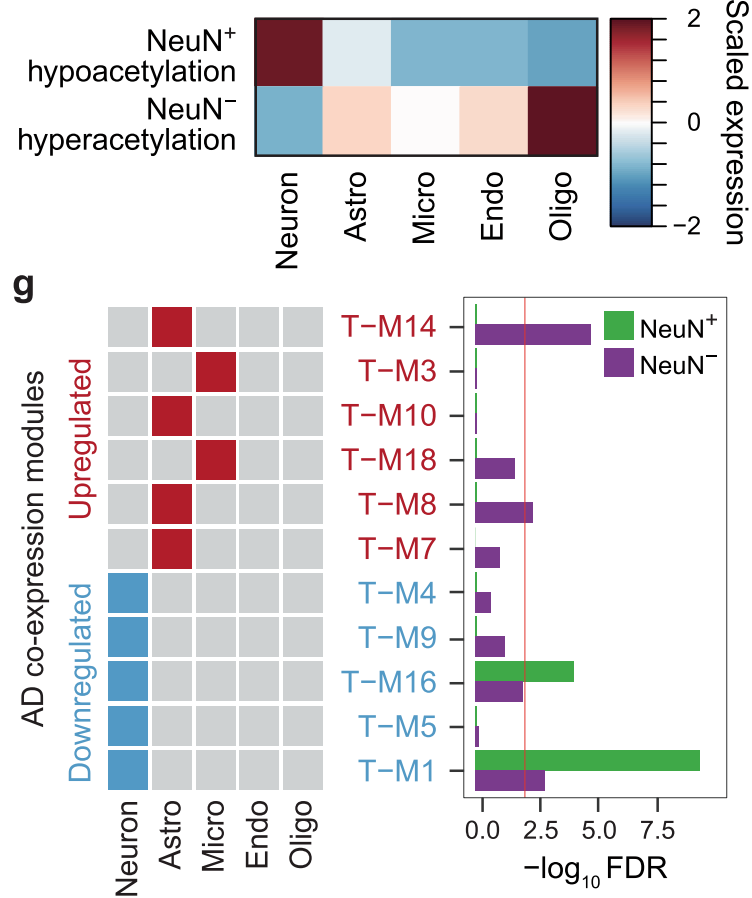

Fig. 3 Cell-type-specific nature of epigenetic dysregulation in AD. a We built AD-associated gene regulatory networks by linking genes to hypoacetylated (hypo) and hyperacetylated (hyper) peaks in AD via $\mathrm{Hi}-\mathrm{C}$ interactions in $\mathrm{NeuN}+$ and $\mathrm{NeuN}-$ cells. $\mathbf{b}$ AD-associated hyperacetylated peaks were largely active in NeuN- cells, while AD-associated hypoacetylated peaks are largely active in NeuN+ cells in neurotypical controls. c The number of genes mapped to AD-associated hyperacetylated (top) and hypoacetylated (bottom) peaks via $\mathrm{Hi}-\mathrm{C}$ interactions in NeuN+ and NeuN- cells. CACNG3 is linked to an AD-associated hypoacetylated peak (marked in yellow) in NeuN+ cells (d), while EHD1 is linked to an AD-associated hyperacetylated peak (marked in yellow) in NeuN - cells (e). CACNG3/EHD1 promoter is highlighted in gray and its interacting regions are highlighted in green and purple for NeuN + and NeuN - cells, respectively. Boxplots in the right show expression levels of CACNG3/EHD1 in NeuN+ $(n=4)$ and NeuN- $(n=4)$ cells. FPKM Fragments Per Kilobase of transcript per Million mapped reads. Center, median; box $=\mathrm{Q} 1-\mathrm{Q} 3$; minima, Q1 - $1.5 \times \mathrm{IQR}$; maxima, Q3+1.5 $\times 1 \mathrm{IQR}$. *FDR $<0.05(\mathrm{FDR}=2.81 \mathrm{e}$ -9 for CACNG3 and FDR $=0.004$ for EHD1), calculated by DESeq2 (two-sided Wald test). Source data are provided as a Source Data file. $\mathbf{f}$ NeuN+ hypoacetylated genes are highly expressed in neurons, while NeuN- hyperacetylated genes are highly expressed in glia. $\mathbf{g}$ NeuN- hyperacetylated genes are enriched in astrocyte-specific co-expression modules (T-M14 and T-M8) that are upregulated in AD. NeuN+ hypoacetylated genes are enriched in neuronal co-expression modules (T-M1, T-M16) that are downregulated in AD. Fisher's exact test was used for statistical analysis. The red line denotes $\mathrm{FDR}=0.01$. Astro astrocytes, Micro microglia, Endo endothelial, Oligo oligodendrocytes.

genes are downregulated due to their hypoacetylation in neurons, whereas many glial genes are upregulated due to their hyperacetylation in glia. To validate this prediction, we tested whether this epigenetic dysregulation in $\mathrm{AD}$ is associated with altered gene expression in $\mathrm{AD}$ by comparing $\mathrm{NeuN}$ - hyperacetylated and $\mathrm{NeuN}+$ hypoacetylated genes with mRNA co-expression modules constructed from AD brains ${ }^{41}$ (Fig. 3g). Among 11 coexpression modules differently regulated in AD (see Supplementary Table 3 for module definition), NeuN- hyperacetylated genes were enriched in co-expression modules that were upregulated in $\mathrm{AD}$ (T-M8 and T-M14) ${ }^{41}$. These modules were involved in transcriptional regulation and cellular proliferation and were annotated as astrocyte-specific ${ }^{41}$. In contrast, NeuN+ hypoacetylated genes were enriched in co-expression modules that were downregulated in $\mathrm{AD}$ (T-M1 and T-M16) ${ }^{41}$. Both modules were associated with synaptic transmission. This result demonstrates that cell-type-specific epigenetic dysregulation in $\mathrm{AD}$ is coupled with gene expression changes. 
a

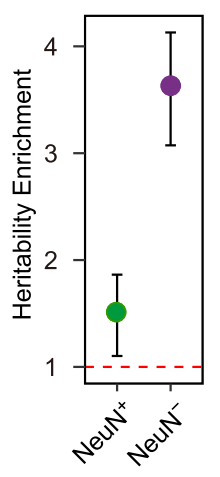

b

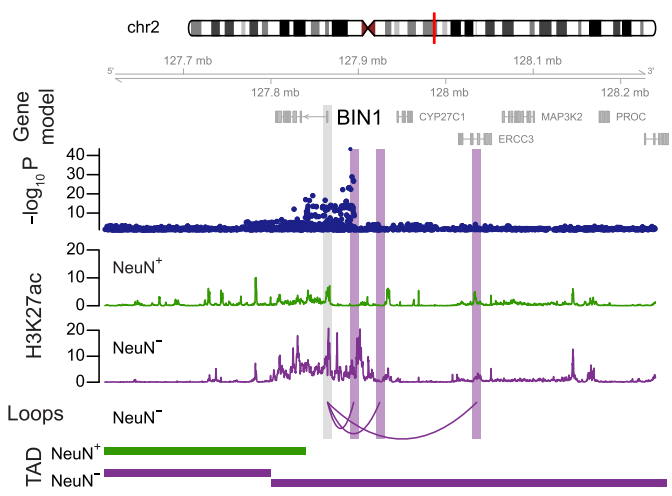

C

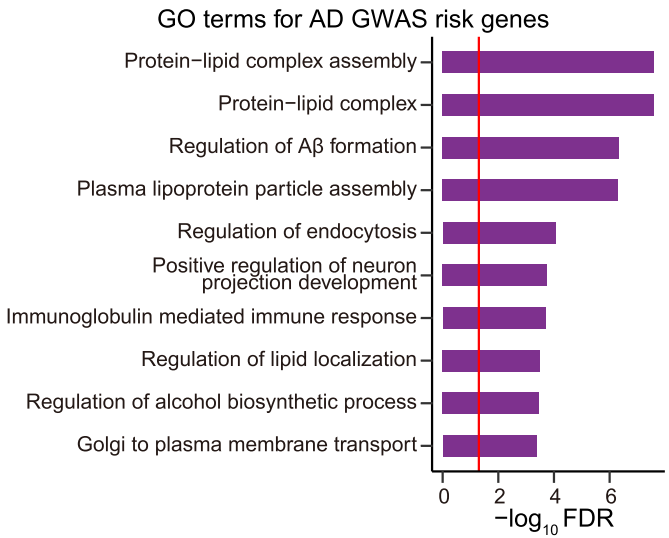

d

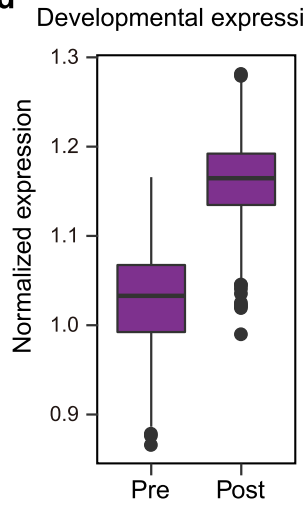

e

Cellular expression

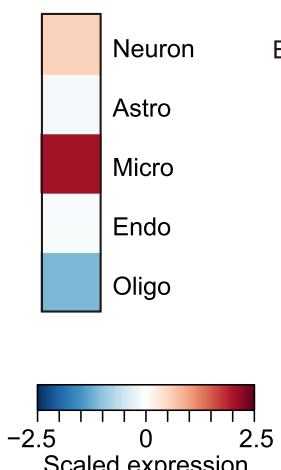

AD scRNA-seq DEG

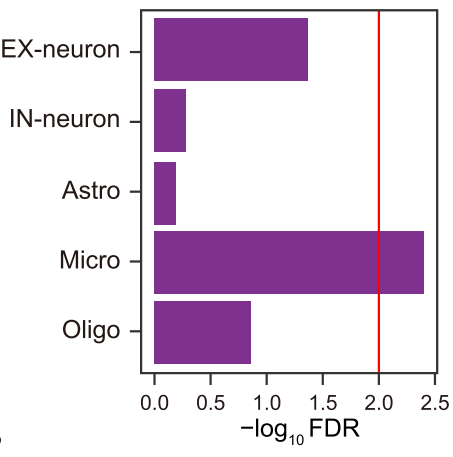

g

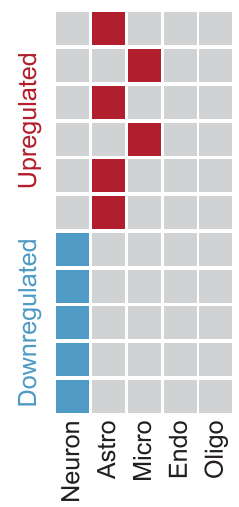

AD modular enrichment

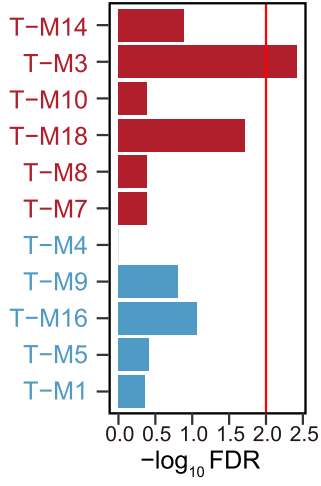

Fig. 4 Identification and characterization of putative target genes of AD genetic risk factors by incorporating NeuN- chromatin interaction. a The SNP-based heritability enrichment of AD GWAS in differential NeuN + and NeuN- peaks suggests glial enrichment. Enrichment \pm standard error is depicted. The red broken line indicates heritability enrichment $=1$. Source data are provided as a Source Data file. b BIN1 promoter physically interacts with an AD GWS locus in a NeuN- specific manner. The regions that interact with BIN1 promoter (marked in gray) are highlighted in purple. c GO analysis for GWASguided AD risk genes identified by NeuN- H-MAGMA. The red line denotes FDR $=0.05$. $\mathbf{d}$ AD risk genes are highly expressed in postnatal brain samples compared with prenatal samples. Pre prenatal $(n=410)$; Post, postnatal $(n=453) . p=6.93 e-193$, calculated by Wilcoxon Rank Sum test. Center, median; box = Q1-Q3; minima, Q1 - 1.5 × IQR; maxima, Q3 + 1.5 × IQR. Source data are provided as a Source Data file. e AD risk genes are highly expressed in microglia. f AD risk genes are significantly enriched for genes differentially regulated in AD microglia. Fisher's exact test was used for statistics analysis. The red line denotes $F D R=0.01$. $\mathrm{g} A D$ risk genes are enriched in a microglial co-expression module that is upregulated in $A D$. Fisher's exact test was used for statistical analysis. The red line denotes FDR $=0.01$. Astro astrocytes, Micro microglia, Endo endothelial, Oligo oligodendrocytes.

Genetic risk factors associated with AD converge onto microglial function. Changes in gene expression or histone modifications may be a consequence or compensatory in disease and not necessarily causal. To link changes in chromatin and gene regulation to causal genetic factors, we next tested for enrichment of common genetic risk factors associated with $\mathrm{AD}$ by $\mathrm{GWAS}^{42}$. We reasoned that cell-type-specific annotation of the regulatory impact of genetic risk factors would provide support or refine our knowledge of causal mechanisms underlying AD, which to date heavily implicate neural immune/microglial mechanisms $s^{43,44}$. Therefore, we first performed linkage disequilibrium score regression (LDSC) ${ }^{45}$ analysis to determine the enrichment of ADassociated common genetic variants in NeuN- and NeuN+ $\mathrm{H} 3 \mathrm{~K} 27 \mathrm{ac}$ peaks. AD SNP-based heritability was highly enriched in $\mathrm{NeuN}-$ enhancers $\left(\mathrm{NeuN}+p=0.205, \mathrm{NeuN}-p=8.41 \times 10^{-9}\right)$, suggesting that $\mathrm{NeuN}-$ gene regulatory relationships would be the most appropriate for refining biological insight from AD GWAS (Fig. 4a).

We next ran H-MAGMA ${ }^{46}$ built upon the NeuN- interactome to convert SNP-level association statistics into gene-level association statistics, thereby connecting non-coding variants to their cognate genes. This analysis identified $181 \mathrm{AD}$ risk genes
$($ FDR $<0.05)$ based upon their potential regulation by associated non-coding variants ("Methods", Supplementary Data 6), many of which included well-known AD risk genes ${ }^{47}$. For instance, we found that $\mathrm{AD}$ genome-wide significant (GWS) SNPs interact with the promoter of $B I N 1$, whose transcript level is increased in AD brains ${ }^{48}$ (Fig. 4b). Notably, the BIN1 promoter was connected to multiple enhancers in $\mathrm{NeuN}-$, but not in $\mathrm{NeuN}+$ cells, demonstrating the highly cell-type-specific gene regulatory landscape within the locus. AD risk genes were enriched for amyloid - beta $(A \beta)$ pathways, lipoprotein assembly, and immune processes (Fig. 4c, Supplementary Data 6). Consistent with previous studies $16,42,49$, these genes were highly expressed in the postnatal brain samples (Fig. 4d) and microglia (Fig. 4e). Notably, they were enriched for genes that are upregulated in microglia from postmortem $\mathrm{AD}$ brain $^{50}$ (Fig. 4f). In line with this, we observed that $\mathrm{AD}$ risk genes that we identified here were enriched for a microglial co-expression module upregulated in $\mathrm{AD}$ postmortem brains (T-M3, Fig. 4g) ${ }^{41}$. This module is distinct from the module associated with epigenetic dysregulation in $\mathrm{AD}$ (Fig. 3g), in that the former represents microglia, while the latter is enriched in astrocytes. This is consistent with recent single-cell sequencing studies identifying genetic risk enrichment and gene 

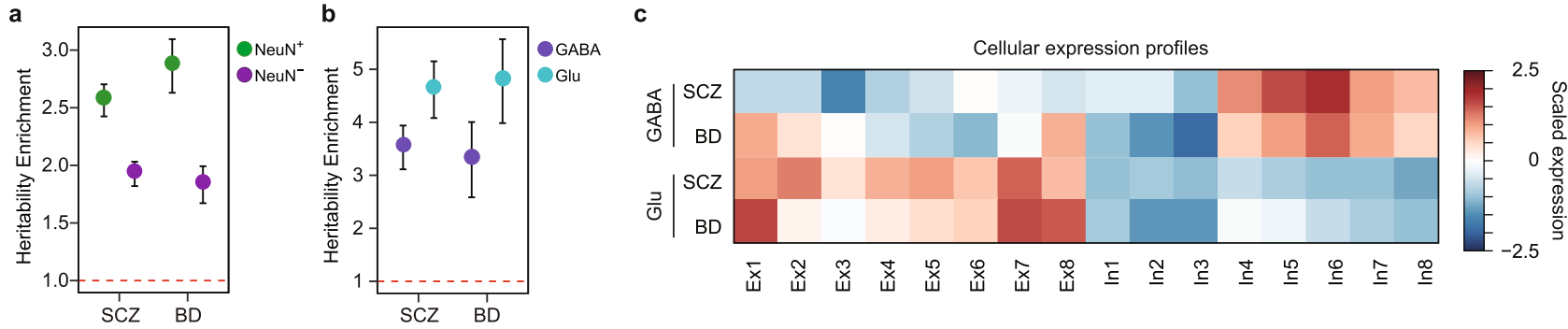

Fig. 5 Comparison of SCZ and BD risk genes. a The SNP-based heritability enrichment of SCZ and BD GWAS in differential NeuN+ and NeuN- peaks demonstrates neuronal enrichment (SCZ NeuN+, FDR = 9.33e-24; SCZ NeuN-, FDR =2.14e-16; BD NeuN+, FDR=3.94e-15; BD NeuN-, FDR=6.09e-07). Enrichment \pm standard error is depicted. The red broken line indicates heritability enrichment $=1$. Source data are provided as a Source Data file. $\mathbf{b}$ The SNPbased heritability enrichment of SCZ and BD GWAS in differential Glu and GABA peaks suggests that both Glu and GABA neurons are associated with the psychiatric disorders (SCZ GABA, FDR $=4.54 \mathrm{e}-9$; SCZ Glu, FDR =3.49e-10; BD GABA, FDR=1.10e-03; BD Glu, FDR =3.63e-06). Enrichment \pm standard error is depicted. The red broken line indicates heritability enrichment $=1$. Source data are provided as a Source Data file. c Neuronal subtype expression profiles of SCZ and BD risk genes detected by GABA and Glu H-MAGMA.

expression changes within microglia in $\mathrm{AD}$, accompanied by significant gene expression changes in other cells, including astrocytes ${ }^{50-52}$, which is where we observe epigenetic signature enrichment. Since genetic risk resides at the initiation of the causal chain in disease pathogenesis, this differential enrichment of genetic risk factors and epigenetic regulation underscores different expression signatures in $\mathrm{AD}$ with distinct cellular specificities and likely causal relationships.

Refined cellular etiology of SCZ and BD. In our previous study, we found that genes associated with psychiatric disorders display substantial molecular convergence within neurons, which was in stark contrast to neurodegenerative disorders like $\mathrm{AD}^{46}$. Indeed, LDSC ("Methods") confirmed that SCZ and BD displayed strong SNP-based heritability enrichment in NeuN+ cells (Fig. 5a). We next hypothesized that enhancer-gene networks at a more refined cellular resolution (e.g. Glu and GABA neurons) would help elaborate molecular processes associated with these two major psychiatric disorders. Therefore, we assessed SNP-based heritability enrichment for SCZ and BD in Glu- and GABA-specific enhancers ${ }^{18}$. Notably, both disorders showed strong enrichment of SNP-based heritability in Glu- and GABA-specific enhancers, suggesting that both excitatory and inhibitory neurons may contribute to the genetic etiology of SCZ and BD (Fig. 5b). Since $\mathrm{Hi}-\mathrm{C}$ data from neuronal subtypes is yet unavailable, we constructed Glu and GABA H-MAGMA maps by integrating Gluand GABA-enhancers with $\mathrm{NeuN}+\mathrm{Hi}-\mathrm{C}$ data, respectively ("Methods"), obtaining 753 SCZ risk genes from Glu (hereby referred to as Glu-SCZ genes) and 624 risk genes from GABAspecific regulatory interactions integrated via H-MAGMA (GABA-SCZ genes) (Supplementary Data 7). Glu-SCZ genes were implicated in synaptic organization, ion channel activity, and neuron projection, while GABA-SCZ genes were associated with dendrite, axon, and hormonal response (Supplementary Data 7). We also identified 143 and $101 \mathrm{BD}$ candidate risk genes from Glu (Glu-BD genes) and GABA (GABA-BD genes) $\mathrm{H}$ MAGMA, respectively (Supplementary Data 7). Glu-BD genes were enriched for neurogenesis, cell adhesion molecules, and synapses, while GABA-BD genes were enriched for transcriptional regulation and NMDA receptor activity (Supplementary Data 7). Neuronal subtype expression profiles of SCZ and BD risk genes identified by Glu and GABA H-MAGMA were evaluated using scRNA data obtained from excitatory (Ex1 to Ex8) and inhibitory (In1 to In8) neuronal subtypes ${ }^{8}$. Neuronal subtype enrichment showed a clear distinction between risk genes identified by Glu and GABA H-MAGMA, uncovering cellular and disease specificities that have not been described before (Fig. 5c).
Glu-SCZ genes displayed widespread expression among many Glu neuronal subclasses, with relatively higher expression signatures in L3/4 neurons (Ex2) and L5/6 corticothalamic projection neurons (Ex7). Glu-BD genes showed a much stronger celltype-specificity, with the highest expression signature in L2/3 cortical projection neurons (Ex1) and L5/6 corticothalamic projection neurons (Ex7-8). In interneurons, GABA-SCZ genes and GABA-BD genes showed similar enrichment for parvalbuminexpressing cells (In6). Given that genetic correlation between SCZ and $\mathrm{BD}$ is remarkably high $(\mathrm{rg}=0.67)^{46,53,54}$, these findings suggest cellular substrates for molecular convergence (Ex7 and In6 are shared between two disorders) and divergence (Ex1 is BD-specific, while Ex2 is SCZ-specific) among two highly genetically correlated disorders.

\section{Discussion}

Here we provide a high-resolution map of chromosome conformation from two major brain cell classes, neurons (NeuN+ cells) and glia (NeuN - cells, Supplementary Fig. 1). The genomewide analysis of chromosome conformation in these two cell classes captures the major known elements of 3D architecture such as compartments, FIREs, and loops, addressing the roles and impact of these different hierarchical units in cell-type-specific gene regulation. We further refined maps of neuronal chromatin architecture by integrating $\mathrm{Hi}-\mathrm{C}$ data from sorted neurons with H3K27ac peaks identified in two major neuronal subtypes, Glu and GABA neurons, which led to a successful delineation of neuronal subtype-specific regulatory relationships. We found that multiple architectural units that include compartments, FIREs, and chromatin loops display a remarked level of cellular specificity that is tightly coupled with gene regulation. We reasoned that these cell-type-specific gene regulatory networks would provide a window through which to understand the cellular etiology of brain diseases.

We first leveraged these networks to deconvolve epigenetic dysregulation in $\mathrm{AD}$ postmortem brain samples into the corresponding cell types. In their original work, Marzi et al. ${ }^{38}$ reported $\mathrm{AD}$-associated $\mathrm{H} 3 \mathrm{~K} 27 \mathrm{ac}$ peaks that are either hypo- or hyperacetylated in AD-affected individuals compared to age-matched low-pathology controls ${ }^{38}$ and linked these peaks to target genes on the basis of linear distance. We and others have shown that enhancers often regulate distal genes ${ }^{4,15,16}$, so this initial assignment is likely to be underpowered and inaccurate. Furthermore, these peaks were obtained from the brain homogenates, which would mask opposing interactions and obscure the cell-typespecific substrate of these changes. By developing neuronal and glial enhancer-promoter interaction maps, we not only accurately 
annotate these peaks with respect to their cognate genes, but also within their corresponding cell types. Notably, AD-associated hyperacetylation was enriched for glial enhancers that are associated with upregulation of astrocytic co-expression modules in $\mathrm{AD}$, while $\mathrm{AD}$-associated hypoacetylation was enriched for neuronal enhancers that potentially affect downregulation of a neuronal co-expression module in $\mathrm{AD}$. These results highlight the importance of obtaining cell-type-specific epigenetic landscapes in diseases. This may reflect either the cellular composition changes (expansion of glia and neuronal death) or changes in regulatory landscape and cellular function (glial activation and decreased neuronal activity) in $\mathrm{AD}$, which requires future investigation.

It is of note that while $\mathrm{AD}$-associated hyperacetylation was linked to oligodendrocyte genes and astrocyte co-expression modules, genetic risk factors for $\mathrm{AD}$ were mapped onto a different glial cell type, microglia. These results are consistent with a newly emerging literature which indicates that different types of glia may contribute to the disease via different regulatory mechanisms ${ }^{55-57}$. In linking $A D$ genetic risk factors to their cognate genes, we also recapitulated a previously found association between rs6733839 and BIN1. Nott et al. found that this SNP is causally implicated in the regulation of an $\mathrm{AD}$ risk gene, $B I N 1$, in a microglia-specific manner ${ }^{16}$ as predicted by our analysis.

In contrast to $\mathrm{AD}$ in which multiple glial cells are implicated, previous work has indicated neurons as the central cell-type harboring genetic risk for the majority of psychiatric disorders $3,22,46,58-61$. However, given the strong genetic overlap between $\mathrm{BD}$ and $\mathrm{SCZ} \mathrm{Z}^{53,62}$, we do not have much indication about specific biological pathways that are driving one disease versus the other. Here, we reasoned that neuronal subtype-specific gene regulatory networks would help refine the cellular etiology of disease and potentially identify discrete molecular neuropathology across psychiatric disorders. Indeed, when we applied Glu and GABA neuronal enhancer-gene networks to $\mathrm{BD}$ and SCZ, we were able to delineate discrete cellular substrates that may contribute to difference between BD (Ex1) and SCZ (Ex5), a distinction that has not been previously recognized. It is also of note that common genetic risk for SCZ and BD also indicated shared pathways coalescing onto parvalbumin-positive interneurons, paralleling what has been among the most robust pathologic findings in $\mathrm{SCZ}$ and $\mathrm{BD}^{63,64}$. However, we note that these findings need to be further verified by neuronal subtype-specific $\mathrm{Hi}-\mathrm{C}$ data (e.g. Glu and GABA Hi-C). Moreover, CRISPR/Cas9mediated genome engineering can be used to target risk genes' enhancers to further investigate their roles in a neuronal subtypespecific manner. Our study provides a foundation for such future study by characterizing cell-type-specific $3 \mathrm{D}$ chromatin structure in the adult human brain, which we show can be used to improve our understanding of gene regulatory landscape in brain disorders.

\section{Methods}

Human tissue collection and nuclei isolation. Prefrontal cortex with no history of neurological disease was procured from tissue collections at the Department of Neuroscience at Yale University School of Medicine. Additional specimens were procured from the Brain and Tissue Bank at the University of Maryland. Tissue was collected after obtaining next of kin consent and with approval by the institutional review boards at the Yale University School of Medicine, the National Institutes of Health, and at each institution from which tissue specimens were obtained. Tissue specimens were handled in accordance with ethical guidelines and regulations for the research use of human brain tissue set forth by the NIH and the WMA Declaration of Helsinki. All available non-identifying information was recorded for each specimen (metadata available in Supplementary Table 1).

Procedures in preparation for flow cytometry (nuclei extraction, NeuN neuronal marker immunotagging using DAPI staining) for frozen never-fixed brain tissue specimens were previously described in detail ${ }^{27,65}$. Briefly, we dissected and homogenized $300 \mathrm{mg}$ of frozen DLPFC tissue in lysis buffer ( $0.32 \mathrm{M}$ Sucrose;
}

$\left.5 \mathrm{mM} \mathrm{CaCl}_{2} ; 3 \mathrm{mM} \mathrm{Mg(Ace)}\right)_{2} ; 0.1 \mathrm{mM}$ EDTA, pH8; $10 \mathrm{mM}$ Tris-HCl, pH8; $1 \mathrm{mM}$ DTT, $0.1 \%$ Triton X-100). Brain homogenate was fixed in $1 \%$ formaldehyde for 10 min prior to NeuN immunotagging ${ }^{66}$. Nuclei were isolated via ultracentrifugation in sucrose gradient. Isolated nuclei were subjected to NeuN immunotagging using 1:1000 anti-NeuN-488 (Millipore, MAB377X) and 1:1000 DAPI (Thermo Fisher Scientific, 62248) diluted in DPBS with $0.2 \%$ BSA. For each $\mathrm{Hi}-\mathrm{C}$ assay, $2-5 \times 10^{6}$ sorted NeuN + or NeuN- nuclei were used. For each nuclear RNA-seq assay, $\sim 5 \times 10^{4}$ sorted nuclei were used.

Hi-C library generation and data processing. Sorted cells were fixed in $1 \%$ formaldehyde for $10 \mathrm{~min}$. Cross-linked DNA was then digested by HindIII (NEB, R0104). Digested chromatin ends were filled, marked with biotin-14-dCTP (Thermo Fisher, 19518-018), and ligated within the nucleus. DNA was sheared into 300-600-bp fragments (Covaris, M220), and biotin-tagged DNA was pulled down with streptavidin beads (Invitrogen, 65001) and ligated with Illumina paired-end adapters. The resulting Hi-C library was amplified by PCR (KAPA Biosystems HiFi HotStart PCR kit, KK2502), and sequenced by Illumina 50 bp paired-end sequencing.

The resulting Hi-C reads were mapped and filtered using hiclib (v.0.9) ${ }^{67}$. Filtered reads were binned at $10 \mathrm{~kb}, 40 \mathrm{~kb}$, and $100 \mathrm{~kb}$ resolution to build a genome-wide contact matrix at a given bin size, which was subsequently normalized using Iterative Correction and Eigenvector decomposition. We then used $100 \mathrm{~kb}$ resolution matrices for compartment analysis, $40 \mathrm{~kb}$ for TAD analysis, and $10 \mathrm{~kb}$ for loop detection. Heatmaps of $\mathrm{Hi}-\mathrm{C}$ contact matrices at $10 \mathrm{~kb}$ resolution were plotted using R package pheatmap (v.1.0.12) with scale="column".

Comparison across multiple brain-derived Hi-C data. We used HiCRep (v.1.10.0) ${ }^{19}$ to measure reproducibility across biological replicates of $\mathrm{Hi}-\mathrm{C}$ libraries using normalized $\mathrm{Hi}-\mathrm{C}$ contact matrices at $40 \mathrm{~kb}$ resolution. HiCRep generates a smoothed contact matrix, and stratifies the matrix by the distance between the interacting regions of chromatin. From this stratified matrix, a SCC is defined, which provides a measure of similarity of genome-wide chromatin contacts across cell types.

HiCRep was also used to compare similarities between brain-derived genomewide normalized chromatin contacts at $40 \mathrm{~kb}$ resolution. We compared $\mathrm{Hi}-\mathrm{C}$ datasets derived from homogenized adult brain tissue (Adult brain) ${ }^{3}$, mature nonneuronal cells $(\mathrm{NeuN}-)$, mature neuronal cells $(\mathrm{NeuN}+)$, postmitotic neuronenriched cortical plate from the fetal brain (Fetal brain CP) ${ }^{4}$, progenitor-enriched ventricular zone from the fetal brain (Fetal brain VZ) ${ }^{4}$, iPSC-derived neurons (iPSC Neuron) $)^{15}$ and iPSC-derived astrocytes (iPSC Astrocytes) ${ }^{15}$.

Compartment calling. HiCExplorer (v.2.2.1.1 $)^{68}$ was used to call compartments from normalized genome-wide chromatin contact matrices at $100 \mathrm{~kb}$ resolution ${ }^{68}$ Principal component analysis (PCA) was performed using 4 eigenvectors. PC values were selected for each chromosome and correlated with gene density to determine compartments. The correlations between PC values from each $\mathrm{Hi}-\mathrm{C}$ data were then used to compare similarity in compartments across cell types.

TAD. We conducted TAD-level analysis as described previously ${ }^{4}$. In brief, we quantified the directionality index by calculating the degree of upstream or downstream $(2 \mathrm{Mb})$ interaction bias of a given bin $(40 \mathrm{~kb})$, which was processed by a hidden Markov model (HMM) to remove hidden directionality bias.

FIRE analysis. We used FIREcaller (v.1.10) ${ }^{24}$ to define FIREs and super-FIREs based on raw $\mathrm{Hi}-\mathrm{C}$ contact matrices at $40 \mathrm{~kb}$ resolution. As FIREcaller defines FIREs based on a specific statistical threshold $(p<0.05$ which corresponds to a FIRE score $>1.56$ ), NeuN+ or NeuN - specific FIREs defined by FIREcaller although displaying similar FIRE scores between two cell types, may be called significant only in one cell, as only one cell type exceeds the statistical cutoff (e.g. FIRE score $=1.6$ ), while the other fails to reach the statistical cutoff (e.g. FIRE score $=1.5$ ). Therefore, rather than using an all-or-none FIRE definition, we identified significantly differential FIREs based on the difference in FIRE scores between NeuN+ and NeuN-. NeuN+ differential FIREs were identified by obtaining the genomic regions that have NeuN + FIRE scores greater than qnorm (0.975) and NeuN- FIRE scores lower than qnorm (0.9). NeuN-differential FIREs were defined in the opposite way; NeuN- FIRE scores<qnorm (0.9) \& NeuN + FIRE scores $>$ qnorm (0.95). To link differential and super-FIREs to target genes, we intersected these differential FIREs and super-FIREs with gene promoters (defined as $2 \mathrm{~kb}$ upstream and $1 \mathrm{~kb}$ downstream of TSS).

We also defined common FIREs, which are FIREs detected in both $\mathrm{NeuN}+$ and NeuN - cells, and the difference of FIREs score between two cell types was less than 0.5 . Common FIREs were then overlapped with promoters to identify common FIRE-associated genes. Since some of the common FIRE-associated genes also overlapped with NeuN + and NeuN- FIREs, we removed those potential cell-typespecific genes to obtain common FIRE-associated genes.

Loop calling. Promoter-based interactions were identified as previously described $^{3,4}$. Briefly, we constructed background interaction profiles from 
randomly selected length- and GC content-matched regions to promoters. Using these background interaction profiles, we fit interaction frequencies into Weibull distribution at each distance for each chromosome using the fitdistrplus ${ }^{69}$ package in R. Significance of interaction from each promoter was calculated as the probability of observing higher interaction frequencies under the fitted Weibull distribution, and interactions with $\mathrm{FDR}<0.01$ were selected as significant promoterbased interactions. We overlapped promoter-based interactions with genomic coordinates of TADs, and found that the majority $(\sim 75 \%)$ of promoter-based interactions were located within the same TADs. We also applied Fit-Hi-C2 $(\text { v.2.0.7 })^{70}$ with $-U 2000000-L 20000-r 10000$ - $p 2$ to call loops.

RNA-seq library generation and data processing. Nuclei (50,000 in DPBS) were sorted into $300 \mu \mathrm{l}$ of Trizol (Fisher; Cat\#: 15596018) such that the volume ratio of nuclei:Trizol was 1:3. Nuclei were lysed by pipetting 20 times in the Trizol solution on ice. RNA extraction was performed using the Zymo Directzol Microprep RNA Kit (Zymo; Cat\#: R2062). RNA quality was evaluated using an Agilent Bioanalyzer 2100 with an RNA 6000 Pico Kit (Agilent; Cat\#5067-1513). The RNA was then converted into cDNA and prepared into RNA-seq libraries using the SmarterStranded kit (Takara; Cat\#634843). The libraries were size selected for an average fragment size of $300 \mathrm{bp}$ using SPRI beads (Beckman Coulter Life Sciences; Cat\#B23317). Library quality was assessed with a Qubit and Agilent Bioanalyzer 2100 using the Agilent High Sensitivity DNA Kit (Cat\#5067-4626).

Once RNA-seq libraries were sequenced, we used FastQC (v.0.11.8) ${ }^{71}$ to check the quality of RNA-seq reads, and removed adapter with Cutadapt program $(\mathrm{v} .1 .18)^{72}$. Next, clean reads were mapped to the human reference genome (Release 19 (GRCh37.p13)) from GENCODE database with HISAT2 (v.2.1.0 $)^{73}$ using default parameters. And, we assembled and quantified transcripts using StringTie $(\mathrm{v} .1 .3 .5)^{74}$. Differential analysis was done with DESeq2 (v.1.22.2 $)^{75}$. RNA-seq data from Glu and GABA neurons were obtained from a previously published study ${ }^{18}$.

ChIP-seq data analysis. Differential H3K27ac peaks between NeuN + and NeuN - cells were obtained from the previously published datasets ${ }^{27}$. We re-analyzed previously published H3K27ac ChIP-seq data from Glu and GABA neurons ${ }^{18}$ to define differential peaks between Glu and GABA neurons. We first used FastQC $(\mathrm{v} .0 .11 .8)^{71}$ to check the quality of ChIP-seq reads ${ }^{18}$. Next, we used Bowtie2 (v.2.3.4.3) ${ }^{76}$ with-very-sensitive to align reads to human reference genome build (Release 19 (GRCh37.p13)) from the GENCODE database. We removed duplicate reads using Picard (v.2.20.1, http://broadinstitute.github.io/picard/) MarkDuplicates function. We then called H3K27ac peaks using MACS2 (v.2.1.0.20150731) ${ }^{77}$ with-broad-cutoff 0.00001 . Finally we used DiffBind (v.2.13.1) ${ }^{78}$ to analyze differentially binding regions between Glu and GABA ChIP-seq data.

Motif analysis. To identify TFs that are involved in cell-type-specific distal regulation, we first extracted differentially accessible chromatin peaks by combining differential $\mathrm{H} 3 \mathrm{~K} 27 \mathrm{ac}^{27}$ and ATAC-seq peaks ${ }^{79}$ that are brought to the promoters via chromatin loops. We then performed differential motif analyses on these celltype-specific distal regulatory peaks using GimmeMotifs (gimme maelstrom) $(\mathrm{v} 0.14 .1)^{30}$ with the default settings.

Gene Ontology Enrichment analysis. We used gProfileR2 (v.0.1.9) ${ }^{80}$ to identify $\mathrm{GO}$ terms that were overrepresented in particular gene sets such as genes assigned to common FIREs, differential FIREs, and super-FIREs. We set the arguments as following:

gost(input,organism $=$ "hsapiens",ordered_query $=\mathrm{F}$,significant $=\mathrm{T}$,evcodes $=$ TRUE, user_threshold $=0.05$, correction_method $=$ "fdr",sources $=c($ (GO")) \$result

Top GO terms for super-FIRE-associated genes were highly redundant, which hindered the identification of biological pathways implicated for super-FIREs. We therefore applied REVIGO with default arguments (http://revigo.irb.hr/) ${ }^{81}$ to remove redundant GO terms.

To analyze functional enrichment of H-MAGMA genes, we used a previously reported rank-based approach ${ }^{46}$. This approach is a threshold-free method, because it does not require a specific FDR cutoff. Instead, biological pathways enriched in the top ranked genes are queried. Briefly, gProfileR (v.0.7.0 $)^{80}$ was used to perform GO analyses for ranked gene lists from H-MAGMA (v.1.08) ${ }^{82}$ for $\mathrm{AD}$, SCZ, and BD GWAS:

gprofiler(input,organism $=$ "hsapiens", ordered_query $=\mathrm{T}$, significant $=\mathrm{T}$, max_p_value $=0.05$, min_set_size $=15$, max_set_size $=600$, min_isect_size $=5$, custom_bg = background gene set, correction_method = "fdr", hier_filtering = "moderate", include_graph $=\mathrm{T}$, src_filter = "GO")

custom_bg: $\mathrm{H}-\mathrm{MAGMA}$ output genes overlapping with $\mathrm{MCH}$ regions were filtered (background gene set);

input: background gene set was ordered based on $p$ value (Ranked gene list) We used gProfileR to perform GO analysis for NeuN+ hypoacetylated and NeuN- hyperacetylated genes:

gprofiler(input,organism $=$ "hsapiens", ordered_query $=F$, significant $=\mathrm{T}$, max_p_value $=0.05$, min_set_size $=15$, min_isect_size $=5$, correction_method="fdr", hier_filtering="moderate", include_graph $=\mathrm{T}$, src_filter="GO").
Cellular expression profile analysis. To quantify the significance of cellular expression of the genes assigned to cell-type-specific chromatin architecture (e.g., differential FIREs and loops), we used EWCE (v.1.3.0) ${ }^{83}$. In addition, cellular expression profiles of the disease risk genes were interrogated by plotting centered expression values for each cell type using the scRNA-seq data as described before ${ }^{10,46}$. Each neuronal subtype definition is provided in Supplementary Table 2

Module enrichment analysis. Developmental and synaptic modules were obtained from Parikshak et al. ${ }^{34}$ and Lips et al. ${ }^{35}$, respectively. We employed Fisher's exact test to compare developmental and synaptic modules with genes engaged in NeuN+ and NeuN- enhancer-promoter interactions. AD-associated co-expression networks were obtained from Seyfried et al. ${ }^{41}$ (Supplementary Table 3).

$\mathrm{AD}$ co-expression modules were compared with (1) genes that were linked to hyper and hypoacetylated genes in $\mathrm{AD}$ and (2) AD-associated genes identified by H-MAGMA ${ }^{46}$ using Fisher's exact test.

Linking AD-associated epigenetic dysregulation to cognate genes. We downloaded AD-associated hyper- and hypoacetylated H3K27ac peaks from Marzi et al. ${ }^{38}$. Because these peaks were obtained from the brain homogenate that lacks cellular resolution, we overlapped them with $\mathrm{NeuN}+$ and $\mathrm{NeuN}-$ differential peaks. Since we found that hyperacetylated peaks in $\mathrm{AD}$ significantly overlapped with NeuN- differential peaks, we used NeuN- loops to assign them to the target genes. On the other hand, hypoacetylated peaks in $\mathrm{AD}$ overlapped with NeuN+ differential peaks, so they were annotated to the target genes by NeuN+ loops.

GWAS data. We downloaded the following GWAS summary datasets: BD $(n=20,352 \text { cases; } 31,538 \text { controls })^{84}$, SCZ $(n=11,260 \text { cases; } 24,542 \text { controls })^{1}$, and $\mathrm{AD}(n=71,880 \text { cases; } 383,378 \text { controls })^{49}$.

LD score regression analysis. We implemented the LDSC software ${ }^{45}$ (v.1.0.0) to estimate the enrichment of SNP-based heritability for brain disorder GWAS in differential H3K27ac peaks between (1) NeuN+ and NeuN- cells and (2) Glu and GABA neurons. Genetic variants were annotated to differential H3K27ac peaks, and SNP-based heritability statistics were calculated using the GWAS summary statistics mentioned above. Enrichment statistics was calculated as the proportion of SNP-based heritability divided by the proportion of SNPs annotated to differential H3K27ac peaks.

H-MAGMA input file generation. We generated H-MAGMA (v.1.08) input files that provide SNP-gene relationships based on chromatin interaction profiles from $\mathrm{NeuN}+$ and NeuN- cells. Exonic and promoter SNPs were directly assigned to their target genes based on their genomic location. Intronic and intergenic SNPs were assigned to their cognate genes based on chromatin interactions with promoters and exons as previously described ${ }^{46}$. To provide SNP-gene relationships at a neuronal subtype resolution, we also generated Glu and GABA H-MAGMA input files. For example, we obtained SNPs that map onto Glu H3K27ac peaks, then SNPs active in Glu neurons were assigned to their genes by either genomic coordinates (when located in promoters and exons) or distal interactions (noncoding SNPs were mapped to their cognate genes via NeuN+ chromatin interactions). We used the same framework to obtain GABA SNP-gene relationships. Input files can be found in the github repository: https://github.com/thewonlab/ NeuN.

H-MAGMA. Based on the SNP-based heritability enrichment result, we used NeuNH-MAGMA to infer AD risk genes and Glu/GABA H-MAGMA to infer SCZ and BD risk genes.

We set the arguments as following: magma_v1.08/magma-bfile g1000_eur - pval $<$ GWAS summary statistics $>$ use $=$ rsid,p ncol $=\mathrm{N}-$ gene-annot $<$ MAGMA input annotation file $>$-out $<$ output file $>$.

g1000_eur denotes the reference data file for European ancestry population.

Python and R environments. We used Python v.2.7 and R v.3.6.0.

Reporting summary. Further information on research design is available in the Nature Research Reporting Summary linked to this article.

\section{Data availability}

Hi-C data described in this manuscript is available through the PsychENCODE Knowledge Portal with the accession code of syn21760712. H3K27ac, ATAC-seq, and RNA-seq data from NeuN+ and NeuN- cells are available through syn4566010, GSE83345, and syn20545534, respectively. H3K27ac ChIP-seq data from Glu and GABA neurons are available through syn12034263. Human reference genome and gene definition was obtained from GENCODE and UCSC genome browser [http://genome. ucsc.edu]. All other relevant data supporting the key findings of this study are available within the article and its Supplementary Information files or from the corresponding 
authors upon reasonable request. Source data are provided with this paper. A reporting summary for this Article is available as a Supplementary Information file. Source data are provided with this paper.

\section{Code availability}

All custom code used in this work is available in the following github repository: https:// github.com/thewonlab/NeuN ${ }^{85}$

Received: 29 April 2020; Accepted: 26 May 2021;

Published online: 25 June 2021

\section{References}

1. Pardiñas, A. F. et al. Common schizophrenia alleles are enriched in mutationintolerant genes and in regions under strong background selection. Nat. Genet. 50, 381-389 (2018).

2. Rada-Iglesias, A. Genetic variation within transcriptional regulatory elements and its implications for human disease. Biol. Chem. 395, 1453-1460 (2014).

3. Wang, D. et al. Comprehensive functional genomic resource and integrative model for the human brain. Science 362, eaat8464 (2018).

4. Won, H. et al. Chromosome conformation elucidates regulatory relationships in developing human brain. Nature 538, 523-527 (2016).

5. de la Torre-Ubieta, L. et al. The dynamic landscape of open chromatin during human cortical neurogenesis. Cell 172, 289-304 (2018).

6. Schmitt, A. D. et al. A compendium of chromatin contact maps reveals spatially active regions in the human genome. Cell Rep. 17, 2042-2059 (2016).

7. Jung, I. et al. A compendium of promoter-centered long-range chromatin interactions in the human genome. Nat. Genet. 51, 1442-1449 (2019).

8. Lake, B. B. et al. Neuronal subtypes and diversity revealed by single-nucleus RNA sequencing of the human brain. Science 352, 1586-1590 (2016)

9. Habib, N. et al. Massively parallel single-nucleus RNA-seq with DroNc-seq. Nat. Methods 14, 955-958 (2017).

10. Darmanis, S. et al. A survey of human brain transcriptome diversity at the single cell level. Proc. Natl Acad. Sci. USA 112, 7285-7290 (2015).

11. Lake, B. B. et al. Integrative single-cell analysis of transcriptional and epigenetic states in the human adult brain. Nat. Biotechnol. 36, 70-80 (2018).

12. Cusanovich, D. A. et al. A single-cell atlas of in vivo mammalian chromatin accessibility. Cell 174, 1309-1324.e18 (2018).

13. Ziffra, R. S. et al. Single cell epigenomic atlas of the developing human brain and organoids. Preprint at bioRxiv https://doi.org/10.1101/2019.12.30.891549 (2020).

14. Trevino, A. E. et al. Chromatin accessibility dynamics in a model of human forebrain development. Science 367, eaay1645 (2020).

15. Rajarajan, P. et al. Neuron-specific signatures in the chromosomal connectome associated with schizophrenia risk. Science 362, eaat4311 (2018).

16. Nott, A. et al. Brain cell type-specific enhancer-promoter interactome maps and disease-risk association. Science 366, 1134-1139 (2019).

17. Matevossian, A. \& Akbarian, S. Neuronal nuclei isolation from human postmortem brain tissue. J. Vis. Exp. https://doi.org/10.3791/914 (2008)

18. Kozlenkov, A. et al. A unique role for DNA (hydroxy) methylation in epigenetic regulation of human inhibitory neurons. Sci. Adv. 4, eaau6190 (2018).

19. Yang, T. et al. HiCRep: assessing the reproducibility of Hi-C data using a stratum-adjusted correlation coefficient. Genome Res. 27, 1939-1949 (2017).

20. $\mathrm{Li}, \mathrm{M}$. et al. Integrative functional genomic analysis of human brain development and neuropsychiatric risks. Science 362, eaat7615 (2018).

21. Halene, T. B. et al. NeuN+ neuronal nuclei in non-human primate prefrontal cortex and subcortical white matter after clozapine exposure. Schizophr. Res. 170, 235-244 (2016).

22. Polioudakis, D. et al. A single-cell transcriptomic atlas of human neocortical development during mid-gestation. Neuron 103, 785-801.e8 (2019).

23. Dixon, J. R. et al. Topological domains in mammalian genomes identified by analysis of chromatin interactions. Nature 485, 376-380 (2012).

24. Crowley, C. et al. FIREcaller: detecting frequently interacting regions from $\mathrm{Hi}-$ C data. Comput. Struct. Biotechnol. J. 19, 355-362 (2021).

25. Halvorsen, M. et al. Increased burden of ultra-rare structural variants localizing to boundaries of topologically associated domains in schizophrenia. Nat. Commun. 11, 1-13 (2020).

26. Gorkin, D. U. et al. Common DNA sequence variation influences 3dimensional conformation of the human genome. Genome Biol. 20, 255 (2019).

27. Girdhar, K. et al. Cell-specific histone modification maps in the human frontal lobe link schizophrenia risk to the neuronal epigenome. Nat. Neurosci. 21, 1126-1136 (2018).
28. Kozlenkov, A. et al. Evolution of regulatory signatures in primate cortical neurons at cell-type resolution. Proc. Natl Acad. Sci. USA 117, 28422-28432 (2020).

29. Consortium, Roadmap Epigenomics. et al. Integrative analysis of 111 reference human epigenomes. Nature 518, 317-330 (2015).

30. van Heeringen, S. J. \& Veenstra, G. J. C. GimmeMotifs: a de novo motif prediction pipeline for ChIP-sequencing experiments. Bioinformatics 27 270-271 (2011).

31. Tamura, T., Yanai, H., Savitsky, D. \& Taniguchi, T. The IRF family transcription factors in immunity and Oncogenesis. Annu. Rev. Immunol. 26 535-584 (2008).

32. Tu, J. C. et al. Homer binds a novel proline-rich motif and links group 1 metabotropic glutamate receptors with IP3 receptors. Neuron 21, 717-726 (1998).

33. Kuhlbrodt, K., Herbarth, B., Sock, E., Hermans-Borgmeyer, I. \& Wegner, M. Sox10, a novel transcriptional modulator in glial cells. J. Neurosci. 18, 237-250 (1998).

34. Parikshak, N. N. N. et al. Integrative functional genomic analyses implicate specific molecular pathways and circuits in autism. Cell 155, 1008-1021 (2013).

35. Lips, E. S. et al. Functional gene group analysis identifies synaptic gene groups as risk factor for schizophrenia. Mol. Psychiatry 17, 996-1006 (2012).

36. Szpirer, C. et al. The genes encoding the glutamate receptor subunits KA1 and KA2 (GRIK4 and GRIK5) are located on separate chromosomes in human, mouse, and rat. Proc. Natl Acad. Sci. USA 91, 11849-11853 (1994).

37. Erlander, M. G., Tillakaratne, N. J., Feldblum, S., Patel, N. \& Tobin, A. J. Two genes encode distinct glutamate decarboxylases. Neuron 7, 91-100 (1991).

38. Marzi, S. J. et al. A histone acetylome-wide association study of Alzheimer's disease identifies disease-associated H3K27ac differences in the entorhinal cortex. Nat. Neurosci. 21, 1618-1627 (2018).

39. Black, J. L. 3rd \& Lennon, V. A. Identification and cloning of putative human neuronal voltage-gated calcium channel gamma-2 and gamma-3 subunits: neurologic implications. Mayo Clin. Proc. 74, 357-361 (1999).

40. Pant, S. et al. AMPH-1/Amphiphysin/Bin1 functions with RME-1/Ehd1 in endocytic recycling. Nat. Cell Biol. 11, 1399-1410 (2009).

41. Seyfried, N. T. et al. A multi-network approach identifies protein-specific coexpression in asymptomatic and symptomatic Alzheimer's disease. Cell Syst. 4, 60-72.e4 (2017)

42. Lambert, J. C. et al. Meta-analysis of 74,046 individuals identifies 11 new susceptibility loci for Alzheimer's disease. Nat. Genet. 45, 1452-1458 (2013)

43. Lee, C. Y. D. \& Landreth, G. E. The role of microglia in amyloid clearance from the AD brain. J. Neural Transm. 117, 949-960 (2010).

44. Novikova, G. et al. Integration of Alzheimer's disease genetics and myeloid genomics identifies disease risk regulatory elements and genes. Nat. Commun. 12, 1610 (2021)

45. Finucane, H. K. et al. Partitioning heritability by functional annotation using genome-wide association summary statistics. Nat. Genet. 47, 1228-1235 (2015).

46. Sey, N. Y. A. et al. A computational tool (H-MAGMA) for improved prediction of brain-disorder risk genes by incorporating brain chromatin interaction profiles. Nat. Neurosci. https://doi.org/10.1038/s41593-020-0603-0 (2020).

47. Matoba, N., Quiroga, I. Y., Phanstiel, D. H. \& Won, H. Mapping Alzheimer's disease variants to their target genes using computational analysis of chromatin configuration. J. Vis. Exp. https://doi.org/10.3791/60428 (2020).

48. Chapuis, J. et al. Increased expression of BIN1 mediates Alzheimer genetic risk by modulating tau pathology. Mol. Psychiatry 18, 1225-1234 (2013).

49. Jansen, I. E. et al. Genome-wide meta-analysis identifies new loci and functional pathways influencing Alzheimer's disease risk. Nat. Genet. 51, 404-413 (2019).

50. Mathys, H. et al. Single-cell transcriptomic analysis of Alzheimer's disease. Nature 570, 332-337 (2019)

51. Grubman, A. et al. A single-cell atlas of entorhinal cortex from individuals with Alzheimer's disease reveals cell-type-specific gene expression regulation. Nat. Neurosci. 22, 2087-2097 (2019).

52. Sala Frigerio, C. et al. The major risk factors for Alzheimer's disease: age, sex, and genes modulate the microglia response to $\mathrm{A} \beta$ plaques. Cell Rep. 27, 1293-1306.e6 (2019).

53. Brainstorm Consortium, The Brainstorm et al. Analysis of shared heritability in common disorders of the brain. Science 360, eaap8757 (2018).

54. Ruderfer, D. M. et al. Polygenic dissection of diagnosis and clinical dimensions of bipolar disorder and schizophrenia. Mol. Psychiatry 19, 1017-1024 (2014).

55. Garwood, C. J., Pooler, A. M., Atherton, J., Hanger, D. P. \& Noble, W. Astrocytes are important mediators of $A \beta$-induced neurotoxicity and tau phosphorylation in primary culture. Cell Death Dis. 2, el67 (2011).

56. Liddelow, S. A. et al. Neurotoxic reactive astrocytes are induced by activated microglia. Nature 541, 481-487 (2017). 
57. Zuchero, J. B. \& Barres, B. A. Glia in mammalian development and disease Development 142, 3805-3809 (2015).

58. Satterstrom, F. K. et al. Large-scale exome sequencing study implicates both developmental and functional changes in the neurobiology of autism. Cell. https://doi.org/10.1016/j.cell.2019.12.036 (2020).

59. Gandal, M. J. et al. Transcriptome-wide isoform-level dysregulation in ASD, schizophrenia, and bipolar disorder. Science 362, eaat8127 (2018).

60. Ruzzo, E. K. et al. Inherited and de novo genetic risk for autism impacts shared networks. Cell 178, 850-866.e26 (2019).

61. Skene, N. G. et al. Genetic identification of brain cell types underlying schizophrenia. Nat. Genet. 50, 825-833 (2018).

62. Cross-Disorder Group of the Psychiatric Genomics Consortium. Genomic relationships, novel loci, and pleiotropic mechanisms across eight psychiatric disorders. Cell 179, 1469-1482.e11 (2019).

63. Enwright Iii, J. F. et al. Transcriptome alterations of prefrontal cortical parvalbumin neurons in schizophrenia. Mol. Psychiatry 23, 1606-1613 (2018).

64. Mellios, N. et al. Molecular determinants of dysregulated GABAergic gene expression in the prefrontal cortex of subjects with schizophrenia. Biol. Psychiatry 65, 1006-1014 (2009).

65. Kundakovic, M. et al. Practical guidelines for high-resolution epigenomic profiling of nucleosomal histones in postmortem human brain tissue. Biol. Psychiatry 81, 162-170 (2017).

66. Espeso-Gil, S. et al. A chromosomal connectome for psychiatric and metabolic risk variants in adult dopaminergic neurons. Genome Med. 12, 19 (2020).

67. Imakaev, $\mathrm{M}$. et al. Iterative correction of $\mathrm{Hi}-\mathrm{C}$ data reveals hallmarks of chromosome organization. Nat. Methods 9, 999-1003 (2012).

68. Ramírez, F. et al. High-resolution TADs reveal DNA sequences underlying genome organization in flies. Nat. Commun. 9, 189 (2018).

69. Delignette-Muller, M. L. et al. fitdistrplus: an R package for fitting distributions. J. Stat. Softw. 64, 1-34 (2015).

70. Kaul, A., Bhattacharyya, S. \& Ay, F. Identifying statistically significant chromatin contacts from Hi-C data with FitHiC2. Nat. Protoc. 15, 991-1012 (2020).

71. Andrews, S. et al. FastQC: a quality control tool for high throughput sequence data (2010).

72. Martin, M. Cutadapt removes adapter sequences from high-throughput sequencing reads. EMBnet. J. 17, 10-12 (2011).

73. Kim, D., Paggi, J. M., Park, C., Bennett, C. \& Salzberg, S. L. Graph-based genome alignment and genotyping with HISAT2 and HISAT-genotype. Nat. Biotechnol. 37, 907-915 (2019).

74. Pertea, M. et al. StringTie enables improved reconstruction of a transcriptome from RNA-seq reads. Nat. Biotechnol. 33, 290-295 (2015).

75. Love, M. I., Huber, W. \& Anders, S. Moderated estimation of fold change and dispersion for RNA-seq data with DESeq2. Genome Biol. 15, 550 (2014).

76. Langmead, B. \& Salzberg, S. L. Fast gapped-read alignment with Bowtie 2. Nat. Methods 9, 357-359 (2012).

77. Zhang, Y. et al. Model-based analysis of ChIP-Seq (MACS). Genome Biol. 9, R137 (2008).

78. Stark, R. et al. DiffBind: differential binding analysis of ChIP-Seq peak data. $R$. Package Version 100, 4-3 (2011).

79. Fullard, J. F. et al. Open chromatin profiling of human postmortem brain infers functional roles for non-coding schizophrenia loci. Hum. Mol. Genet. 26, 1942-1951 (2017).

80. Raudvere, U. et al. g:Profiler: a web server for functional enrichment analysis and conversions of gene lists (2019 update). Nucleic Acids Res. R Package Version 0. 6 47, W191-W198 (2019).

81. Supek, F., Bošnjak, M., Škunca, N. \& Šmuc, T. REVIGO summarizes and visualizes long lists of gene ontology terms. PLoS ONE 6, e21800 (2011).

82. de Leeuw, C., Sey, N. Y. A., Posthuma, D. \& Won, H. A response to Yurko et al: H-MAGMA, inheriting a shaky statistical foundation, yields excess false positives. https://doi.org/10.1101/2020.09.25.310722 (2020).

83. Skene, N. G. \& Grant, S. G. N. Identification of vulnerable cell types in major brain disorders using single cell transcriptomes and expression weighted cell type enrichment. Front. Neurosci. 10, 16 (2016).

84. Stahl, E. A. et al. Genome-wide association study identifies 30 loci associated with bipolar disorder. Nat. Genet. 51, 793-803 (2019).
85. Hu, B. \& hyejungwon. thewonlab/NeuN v1.0.0. https://doi.org/10.5281/ zenodo.4552591 (2021)

\section{Acknowledgements}

We thank members of the Won lab for helpful discussions and comments about this paper We also thank Sergio Espeso Gil for transferring RNA-seq datasets from NeuN+ and NeuNcells. This research was supported by the National Institute of Health (NIMH: P50MH106438, R01MH094714, U01MH103339, R01MH110927, R01MH100027, D.H.G.; R00MH113823, DP2MH122403, R21DA051921, R01AG066871, H.W.; U01MH103392, S.A.; R21MH103877, U01MH122590, R01DA044940, S.D.), the Veterans Administration Biomedical Laboratory Research \& Development (I01BX002876, S.D.), a NARSAD Young Investigator Award from the Brain and Behavior Research Foundation (H.W.), a SPARK grant from the Simons Foundation Autism Research Initiative (H.W.), and Helen Lyng White Fellowship (W.M.). We also acknowledge the support from the Data Science Core at the UNC IDDRC (P50HD103573). The DLPFC tissue used in this research was obtained from the Human Brain Collection Core, Intramural Research Program, NIMH (http://www.nimh.nih.gov/ $\mathrm{hbcc}$. We thank Drs. Stefano Marenco, Barbara Lipska, and Pavan Auluck for their assistance in tissue procurement.

\section{Author contributions}

The design of the NeuN+ and NeuN- Hi-C comparison was developed as part of a reference brain map for the PsychENCODE consortium by D.H.G., S.A., and N.S. Tissue dissection and dissemination has been conducted by S.P. and N.S. Cells were sorted from the adult DLPFC to neurons $(\mathrm{NeuN}+$ ) and glia (NeuN-) by R.B.P., B.K., and S.A. The subsequent integrative analysis was designed by H.W. and D.H.G. B.H. performed overall data analyses. K.S. ran HiCRep analyses. S.A. contributed H3K27ac and RNA-seq data from NeuN + and NeuN- cells. A.K. and S.D. contributed H3K27ac data from glutamatergic and GABAergic neurons. C.A.C. and Y.L. helped FIRE analyses. B.H. and W.M. generated and edited figures. B.H. and H.W. co-wrote the first draft of the manuscript, which was subsequently revised by D.H.G., S.A., and S.D.

\section{Competing interests}

The authors declare no competing interests.

\section{Additional information}

Supplementary information The online version contains supplementary material available at https://doi.org/10.1038/s41467-021-24243-0.

Correspondence and requests for materials should be addressed to H.W. or D.H.G.

Peer review information Nature Communications thanks the anonymous reviewer(s) for their contribution to the peer review of this work.

Reprints and permission information is available at http://www.nature.com/reprints

Publisher's note Springer Nature remains neutral with regard to jurisdictional claims in published maps and institutional affiliations.

cc) (i) Open Access This article is licensed under a Creative Commons BY Attribution 4.0 International License, which permits use, sharing adaptation, distribution and reproduction in any medium or format, as long as you give appropriate credit to the original author(s) and the source, provide a link to the Creative Commons license, and indicate if changes were made. The images or other third party material in this article are included in the article's Creative Commons license, unless indicated otherwise in a credit line to the material. If material is not included in the article's Creative Commons license and your intended use is not permitted by statutory regulation or exceeds the permitted use, you will need to obtain permission directly from the copyright holder. To view a copy of this license, visit http://creativecommons.org/ licenses/by/4.0/.

(c) The Author(s) 2021 


\section{The PsychENCODE Consortium}

Allison E. Ashley-Koch ${ }^{17}$, Gregory E. Crawford ${ }^{17}$, Melanie E. Garrett ${ }^{17}$, Lingyun Song ${ }^{17}$, Alexias Safi ${ }^{17}$, Graham D. Johnson ${ }^{17}$, Gregory A. Wray ${ }^{17}$, Timothy E. Reddy ${ }^{17}$, Fernando S. Goes ${ }^{18}$, Peter Zandi ${ }^{18}$, Julien Bryois ${ }^{19}$, Andrew E. Jaffe 20 , Amanda J. Price 20 , Nikolay A. Ivanov ${ }^{20}$, Leonardo Collado-Torres ${ }^{20}$, Thomas M. Hyde ${ }^{20}$, Emily E. Burke ${ }^{20}$, Joel E. Kleiman20, Ran Tao ${ }^{20}$, Joo Heon Shin20, Kiran Girdhar ${ }^{3,4}$, Yan Jiang ${ }^{3,4}$, Marija Kundakovic 3,4, Leanne Brown ${ }^{3,4}$, Jennifer R. Wiseman ${ }^{3,4}$, Elizabeth Zharovsky ${ }^{3,4}$, Rivka Jacobov ${ }^{3,4}$, Olivia Devillers 3,4 , Elie Flatow ${ }^{3,4}$, Gabriel E. Hoffman ${ }^{3,4}$, Judson Belmont ${ }^{3,4}$, Diane Del Valle ${ }^{3,4}$, Nancy Francoeur 3,4, Evi Hadjimichael ${ }^{3,4}$, Dalila Pinto 3,4 , Harm van Bakel ${ }^{3,4}$, Panos Roussos 3,4, John F. Fullard ${ }^{3,4}$, Jaroslav Bend1 ${ }^{3,4}$, Mads E. Hauberg ${ }^{3,4}$, Alexander W. Charney ${ }^{3,4}$, Vahram Haroutunian ${ }^{5}$, Barbara K. Lipska ${ }^{21}$, David A. Lewis ${ }^{22}$, Chang-Gyu Hahn ${ }^{23}$, Lara M. Mangravite ${ }^{24}$, Mette A. Peters ${ }^{24}$, Yooree Chae 24 , Junmin Peng ${ }^{25}$, Mingming Niu ${ }^{25}$, Xusheng Wang ${ }^{25}$, Maree J. Webster ${ }^{26}$, Thomas G. Beach ${ }^{27}$, Chao Chen ${ }^{28}$, Yi Jiang ${ }^{28,}$ Rujia Dai ${ }^{28}$, Yongjun Wang 28 , Yan Xia ${ }^{28,29}$, Annie W. Shieh ${ }^{29}$, Chunyu Liu ${ }^{29}$, Kay S. Grennan ${ }^{29}$, Ramu Vadukapuram ${ }^{29}$, Gina Giase 29 , Dominic Fitzgerald ${ }^{30}$, Lijun Cheng 30 , Miguel Brown ${ }^{30}$, Mimi Brown ${ }^{30}$, Tonya Brunetti ${ }^{30}$, Thomas Goodman ${ }^{30}$, Majd Alsayed ${ }^{30}$, Kevin P. White ${ }^{30}$, Mohana Ray ${ }^{30}$, Damon Polioudakis ${ }^{13}$, Brie Wamsley ${ }^{13}$, Jiani Yin ${ }^{13}$, Luis De La Torre Ubieta ${ }^{13}$, Michael J. Gandal ${ }^{13}$, Vivek Swarup ${ }^{13}$, Stephan J. Sanders ${ }^{31}$, Matthew W. State ${ }^{31}$, Donna M. Werling ${ }^{31}$, Joon-Yong An ${ }^{31}$, Brooke Sheppard ${ }^{31}$, A. Jeremy Willsey ${ }^{31}$, Amira Kefi ${ }^{32}$, Eugenio Mattei ${ }^{33}$, Michael Purcaro ${ }^{33}$, Zhiping Weng ${ }^{33}$, Jill Moore ${ }^{33}$, Henry Pratt ${ }^{33}$, Jack Huey ${ }^{33}$, Tyler Borrman ${ }^{33}$, Patrick F. Sullivan², Paola Giusti-Rodriguez², Yunjung Kim², Jin Szatkiewicz², Suhn Kyong Rhie ${ }^{34}$, Christoper Armoskus ${ }^{34}$, Adrian Camarena ${ }^{34}$, Peggy J. Farnham ${ }^{34}$, Valeria N. Spitsyna ${ }^{34}$, Heather Witt ${ }^{34}$, Shannon Schreiner ${ }^{34}$, Oleg V. Evgrafov ${ }^{35}$, James A. Knowles ${ }^{35}$, Mark Gerstein ${ }^{36}$, Shuang Liü ${ }^{36}$, Fabio C. P. Navarro ${ }^{36}$, Jonathan Warrell ${ }^{36}$, Declan Clarke ${ }^{36}$, Prashant S. Emani ${ }^{36}$, Mengting Gu ${ }^{36}, \mathrm{Xu} \mathrm{Shi}^{36}$, Min Xu ${ }^{36}$, Yucheng T. Yang ${ }^{36}$, Robert R. Kitchen ${ }^{36}$, Gamze Gürsoy ${ }^{36}$, Jing Zhang ${ }^{36}$, Becky C. Carlyle ${ }^{6}$, Angus C. Nairn' ${ }^{6}$, Mingfeng Li ${ }^{6}$, Mario Skarica ${ }^{6}$, Zhen Li ${ }^{6}$, Andre M. M. Sousa ${ }^{6}$, Gabriel Santpere ${ }^{6}$, Jinmyung Choi ${ }^{6}$, Ying Zhu ${ }^{6}$, Tianliuyun Gao ${ }^{6}$, Daniel J. Miller ${ }^{6}$, Adriana Cherskov ${ }^{6}$, Mo Yang ${ }^{6}$, Anahita Amiri ${ }^{6}$, Gianfilippo Coppola ${ }^{6}$, Jessica Mariani ${ }^{6}$, Soraya Scuderi ${ }^{6}$, Anna Szekely ${ }^{6}$, Flora M. Vaccarino ${ }^{6}$, Feinan Wu ${ }^{6}$, Sherman Weissman' ${ }^{6}$, Daifeng Wang ${ }^{37}$, Tanmoy Roychowdhury ${ }^{38}$ \& Alexej Abyzov ${ }^{38}$

\footnotetext{
${ }^{17}$ Duke University, Durham, NC, USA. ${ }^{18}$ Johns Hopkins University, Baltimore, MD, USA. ${ }^{19}$ Karolinska Institutet, Stockholm, Sweden. ${ }^{20}$ Lieber Institute for Brain Development, Baltimore, MD, USA. ${ }^{21}$ Human Brain Collection Core, National Institutes of Health, Bethesda, MD, USA. ${ }^{22}$ University of Pittsburgh, Pittsburg, PA, USA. ${ }^{23}$ University of Pennsylvania, Philadelphia, PA, USA. ${ }^{24}$ Sage Bionetworks, Seattle, WA, USA. ${ }^{25}$ St. Jude Children's Hospital, Memphis, TN, USA. ${ }^{26}$ Stanley Medical Research Institute, Kensington, MD, USA. ${ }^{27}$ Banner Sun Health Research Institute, Sun City, AZ, USA. ${ }^{28}$ Central South University, Changsha, Hunan, China. ${ }^{29}$ SUNY Upstate Medical University, Syracuse, NY, USA. ${ }^{30}$ The University of Chicago, Chicago, IL, USA. ${ }^{31}$ University of California-San Francisco, San Francisco, CA, USA. ${ }^{32}$ University of Illinois at Chicago, Chicago, IL, USA. ${ }^{33}$ University of Massachusetts Medical School, Worcester, MA, USA. ${ }^{34}$ University of Southern California, Los Angeles, CA, USA. ${ }^{35}$ SUNY Downstate Medical Center, Brooklyn, NY, USA. ${ }^{36}$ Program in Computational Biology and Bioinformatics, Yale University, New Haven, CT, USA.

${ }^{37}$ University of Wisconsin-Madison, Madison, WI, USA. ${ }^{38}$ Mayo Clinic Rochester, Rochester, MN, USA.
} 